\title{
Combined SFK/MEK inhibition prevents metastatic outgrowth of dormant tumor cells
}

\author{
Lara H. El Touny, ${ }^{1}$ Anthony Vieira, ${ }^{1}$ Arnulfo Mendoza, ${ }^{2}$ Chand Khanna, ${ }^{2}$ \\ Mark J. Hoenerhoff, ${ }^{3}$ and Jeffrey E. Green ${ }^{1}$
}

\author{
1'Laboratory of Cancer Biology and Genetics and 2Pediatric Oncology Branch, National Cancer Institute, Bethesda, Maryland, USA. \\ ${ }^{3}$ Cellular and Molecular Pathology Branch, National Toxicology Program, \\ National Institute of Environmental Health Sciences, Research Triangle Park, North Carolina, USA.
}

\begin{abstract}
Breast cancer $(\mathrm{BC})$ can recur as metastatic disease many years after primary tumor removal, suggesting that disseminated tumor cells survive for extended periods in a dormant state that is refractory to conventional therapies. We have previously shown that altering the tumor microenvironment through fibrosis with collagen and fibronectin deposition can trigger tumor cells to switch from a dormant to a proliferative state. Here, we used an in vivo preclinical model and a 3D in vitro model of dormancy to evaluate the role of Src family kinase (SFK) in regulating this dormant-to-proliferative switch. We found that pharmacological inhibition of SFK signaling or $S r c$ knockdown results in the nuclear localization of cyclin-dependent kinase inhibitor $\mathrm{p} 27$ and prevents the proliferative outbreak of dormant BC cells and metastatic lesion formation; however, SFK inhibition did not kill dormant cells. Dormant cell proliferation also required ERK1/2 activation. Combination treatment of cells undergoing the dormant-to-proliferative switch with the Src inhibitor (AZD0530) and MEK1/2 inhibitor (AZD6244) induced apoptosis in a large fraction of the dormant cells and delayed metastatic outgrowth, neither of which was observed with either inhibitor alone. Thus, targeting Src prevents the proliferative response of dormant cells to external stimuli, but requires MEK1/2 inhibition to suppress their survival. These data indicate that treatments targeting Src in combination with MEK1/2 may prevent $\mathrm{BC}$ recurrence.
\end{abstract}

\section{Introduction}

The recurrence of breast cancer (BC) as a disseminated disease remains the second major cause of cancer mortality in women in the United States (1). The recognition that tumor cells may disseminate at very early stages of BC (2) and that metastatic disease may recur many years after initial therapy strongly suggests that disseminated cells can survive for extended periods in a growth-arrested state (3).

Tumor dormancy may exist in several biologically distinct manifestations. Individual quiescent tumor cells have been found in the bone marrow of patients and potentially proliferate in response to stimuli or additional genetic alterations (4). Autopsy studies have demonstrated the presence of micrometastases without clinical disease whose growth may be suppressed by a lack of angiogenic signaling or kept in check through immune surveillance (5). Understanding what regulates the dormant-to-proliferative switch of latent tumor cells may lead to new approaches for preventing recurrent disease.

The microenvironment plays a critical role in breast tumorigenesis and metastasis with the extracellular matrix (ECM) exerting a critical influence on these processes (6-8). We previously used a well-characterized model of mammary tumor cell dormancy whereby related cell lines derived from spontaneous mammary hyperplastic alveolar nodules exhibited either a proliferative (D2A1 cells) or dormant (D2.0R cells) phenotype at metastatic sites (9). Our group demonstrated that an in vitro 3D culture system was predictive of dormant or proliferative behavior of human $\mathrm{BC}$ cell lines and that the addition of collagen 1 (COL1) or fibronectin, ECM components associated with fibrosis and

Conflict of interest: The authors have declared that no conflict of interest exists. Citation for this article: J Clin Invest. 2014;124(1):156-168. doi:10.1172/JCI70259. tumorigenesis, could induce the proliferation of otherwise quiescent D2.0R cells $(10,11)$. Additionally, by inducing fibrosis at the lung metastatic site, otherwise dormant cells would proliferate into large, metastatic outgrowths (11). The induction of the dormant-to-proliferative switch required activation of the integrin $\beta 1$ (ITGB1) receptor and signaling through the activation of focal adhesion kinase (FAK), Src, ERK1/2, and MLCK, leading to actin stress fiber formation $(10,11)$.

Based on our previous observations that Src and the mitogen-activated protein kinase (ERK/MAPK) are required for the dormant-to-proliferative switch, we hypothesized that these might be potential targets for preventing tumor recurrence in a preclinical setting. Src activity is required for integrin-dependent signaling events (12) and its expression has been closely associated with BC metastasis, increased risk of bone metastases, and poor progression-free survival in $\mathrm{BC}$ patients $(13,14)$. Src activation has also been shown experimentally to be required for the establishment of bone and lung metastases by enhancing cell survival and proliferation of metastatic lesions $(15,16)$. Saracatinib (AZD0530; AstraZeneca) is an orally active, dual Src family kinase-AB1 (SFK$A B L$ ) inhibitor that prevents Src-associated signaling (17) and is currently being tested in phase II clinical trials.

The MAPK pathway is activated downstream of integrin signaling (18). Upregulation of ERK/MAPK is associated with an increased risk of tumor recurrence and reduced survival in patients with triple-negative BC (19). ERK/MAPK activation occurred in pulmonary metastases in a murine BC model (20), suggesting a positive role for ERK/MAPK in the establishment of pulmonary metastases. Selumetinib, also known as AZD6244 or ARRY-142886 (AstraZeneca) is a potent, selective, noncompetitive ATP inhibitor of kinases MEK1/2 that specifically activates ERK/ MAPK and is currently in phase II clinical development (21). 
In this study, we explored the potential therapeutic application of SFK and MEK1/2 inhibitors on the dormant-to-proliferative process of metastatic progression using our established in vitro and in vivo models of cancer cell dormancy. We demonstrate that Src inhibition by AZD0530 or shRNA knockdown in 3D culture prevents the COL1-induced proliferation of dormant tumor cells and is associated with the upregulation and nuclear localization of the cell cycle inhibitor p27. However, the effect of Src inhibition on proliferation in vitro is not sustained once the SFK inhibitor is withdrawn. Additionally, the SFK inhibitor has a minimal effect on the growth of metastatic lesions once the dormant cells have transitioned to proliferative lesions. Thus, Src inhibition maintains quiescence, but does not revert proliferating cells to a dormant state or induce cell death. Although ERK1/2 activation by COL1 engagement with ITGB1 is not affected by Src inhibition, inhibiting ERK1/2 in combination with the SFK inhibitor AZD0530 results in the apoptotic death of dormant cells, which does not occur using either agent alone. These results suggest that the combination of an SFK inhibitor and an MEK1/2 inhibitor may represent a new strategy for decreasing the burden of disseminated metastatic dormant cells and preventing disease recurrence.

\section{Results}

SFK inhibition prevents the COL1-induced dormant-to-proliferative switch of D2.0R cells. We have previously shown that COL1 in a 3D basement membrane extract (BME) culture system induces the proliferation of otherwise dormant D2.0R cells through an Src-dependent integrin $\beta 1$ signaling cascade (11). In this study, we examined the effect of Src inhibition on maintaining tumor dormancy and reducing metastatic growth in a preclinical setting. D2.0R cells on BME plus COL1 were seeded and immediately treated with a range of AZD0530 doses in a growth prevention regimen. We observed a dose-dependent reduction in proliferation, with complete inhibition of growth observed at a dose of $250 \mathrm{nM}$ AZD0530 on BME plus COL1, which is consistent with serum levels obtained in clinical trials (refs. 22, 23, and Figure 1A). Importantly, we observed the inhibition of proliferation by AZD0530 treatment as early as 24 hours after treatment, with no further increase in cell numbers over time, but with continued cell viability (Supplemental Figure 1A; supplemental material available online with this article; doi:10.1172/JCI70259DS1). The AZD0530-mediated reduction in proliferation was accompanied by an $85 \%$ reduction in phosphorylated Src ( $\left.\mathrm{pSrc} ; \mathrm{Y}^{416}\right)$, an indicator of Src activity, as well as a reduction in the Src target $\mathrm{PFAK}\left(\mathrm{Y}^{576 / 577}\right)$ (Figure 1B), with no effect on total Src or FAK expression.

Since AZD0530 is an SFK inhibitor, we determined whether other Src family members are expressed by D2.0R cells grown on BME plus COL1 and found only c-YES to be expressed at levels comparable to those of Src at the mRNA and protein levels (Supplemental Figure 1B). In order to confirm that Src inhibition by AZD0530 is specifically responsible for the observed inhibition of proliferation of D2.0R cells, we established stable cell lines expressing nontarget (NT) shRNA or Src shRNA (Figure 1C). Clones 3 and 4 exhibited a $62 \%$ and $70 \%$ knockdown of Src expression, respectively, compared with that observed in parental and NT shRNA cells, with no effect on c-YES levels (Supplemental Figure 1C). These clones failed to proliferate on BME plus COL1 (Figure 1D), although they remained viable, as determined by calcein-AM staining (Supplemental Figure 1A). Clones 1 and 2, with a less substantial knockdown of Src protein levels, were still able to proliferate but at a reduced rate compared with parental and shNT cells (Figure 1D). Furthermore, Src inhibition in D2.0R cells by shRNA or AZD0530 did not result in a significant reduction in cell numbers on BME without COL1 (Figure 1, E and F). This suggests that SFK inhibition is only effective at inhibiting the dormant-to-proliferative switch mediated by COL1 supplementation without any substantial effect on the viability of dormant cells seeded on BME alone.

SFK inbibition prevents the fibrosis-induced dormant-to-proliferative switch. Given the in vitro findings related to SFK inhibition described above, we examined the effectiveness of AZD0530 on modulating dormancy in our previously reported in vivo model of a fibrosis-induced dormant-to-proliferative switch of D2.0R cells (11). The induction of lung fibrosis in CD $1^{n u / n u}$ mice via adenoTGF- $\beta^{223 / 225}$ followed 3 weeks later by tail-vein injection of D2.0R GFP cells resulted in a significant 20 -fold increase in the average total metastatic burden, as measured by quantitative fluorescence of metastatic cells in the lung $\left(160,000 \pm 49,000\right.$ pixels $\left.^{2}\right)$ as compared with the metastatic burden observed when the same cells were tail-vein injected into CD $1^{n u / n u}$ mice with nonfibrotic lungs $\left(7,640 \pm 1,950\right.$ pixels $\left.^{2}\right)(P=0.02)$ (Figure $\left.2 \mathrm{~A}\right)$. We have previously demonstrated that a pixels ${ }^{2}$ count of an individual lesion of less than 1,000 corresponds to a single, dormant tumor cell, whereas lesions greater than 1,000 pixels $^{2}$ represent proliferative, metastatic lesions $(10,11)$. Fibrotic lungs exhibited a 5 -fold increase in the percentage of proliferative metastatic lesions in the lungs compared with nonfibrotic lungs (Figure 2B). However, the daily administration of AZD0530 (50 mg/kg body weight) by gavage beginning 24 hours after tail-vein injection of D2.0R GFP cells resulted in an 8-fold reduction in the average metastatic burden in fibrotic lungs and abrogated the increased percentage of proliferative lesions to levels seen in nonfibrotic lungs (Figure 2, A and B) without affecting fibrosis-induced collagen deposition in the lungs (Supplemental Figure 2A). Consistent with the in vitro results described above, the administration of AZD0530 to CD $1^{n u / n u}$ mice instilled with empty adenovirus (Ad-empty; no fibrosis) did not significantly modulate the metastatic burden or the percentages of dormant versus proliferative lesions (Figure 2, A and B).

Genetic knockdown of Src inhibits the dormant-to-proliferative switch. To further demonstrate the critical role of Src in the fibrosis-induced dormant-to-proliferative switch, we performed in vivo experiments using shNT or shSrc D2.0R cells (clones 3 and 4). We observed a significant increase $(P=0.0157)$ in total metastatic burden in fibrotic versus nonfibrotic lungs with control shNT D2.0R cells, similar to that observed in parental D2.0R cells (Figure 2C). In contrast, shSrc D2.0R cells resulted in an approximately 10 - to 15 -fold reduction in total lung metastatic burden (Figure 2C). Importantly, the percentage of dormant cells versus proliferative lesions in fibrotic lungs with the shSrc D2.0R clones was similar to that observed when shNT D2.0R cells were injected into mice without lung fibrosis ( $~ 80 \%$ dormant cells and $20 \%$ proliferative lesions) (Figure 2D), demonstrating that shSrc $\mathrm{D} 2.0 \mathrm{R}$ cells were unable to undergo the dormant-to-proliferative switch in fibrotic lungs. This further underscores the importance of Src activity in the proliferation of dormant cells in the context of a fibrotic environment.

Analysis of the effect of SFK inhibition on the dormant-to-proliferative switch using an ex vivo lung metastasis assay. We examined the effects of SFK inhibition on the dormant-to-proliferative switch using an ex vivo pulmonary metastatic assay (PuMA) to observe the behavior of metastatic cells in real time in lung culture sections. In this 
A

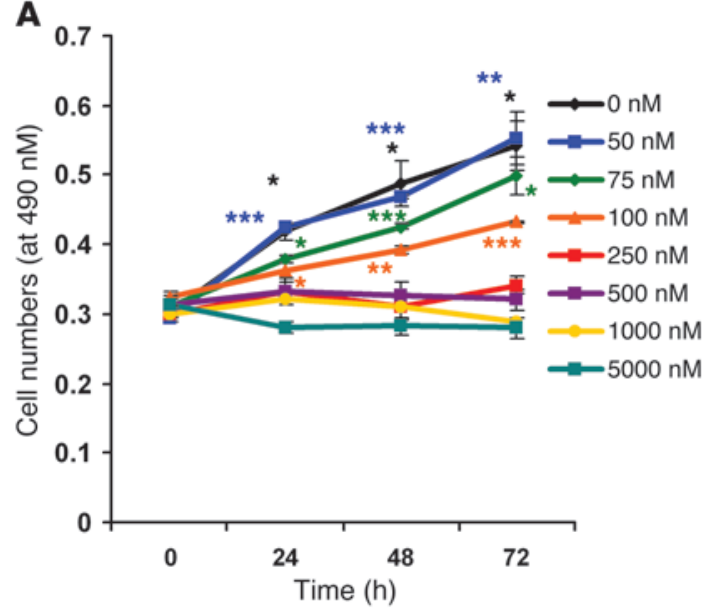

B

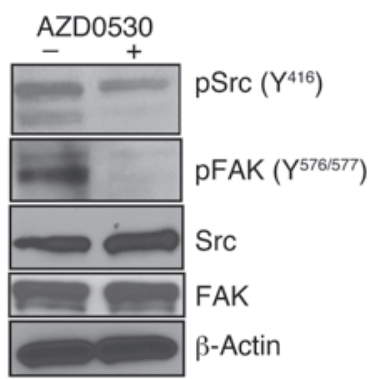

C

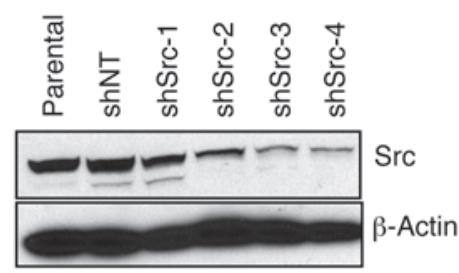

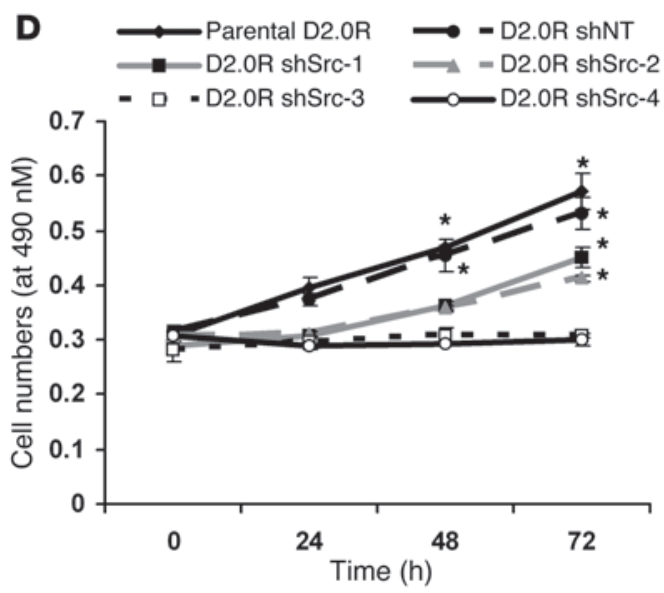
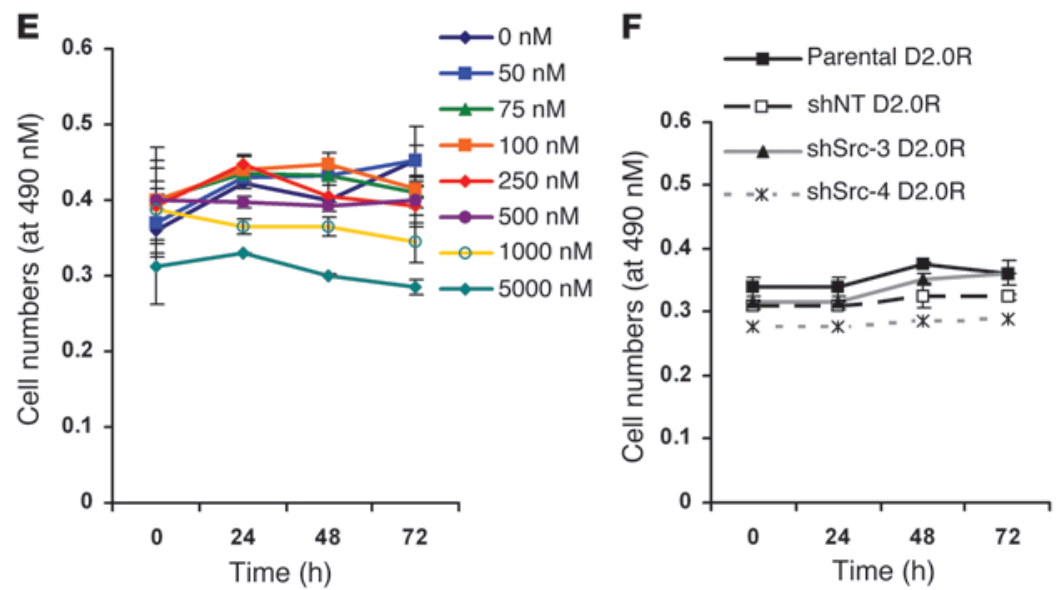

Figure 1

SFK inhibition prevents the dormant-to-proliferative switch of D2.0R cells on BME plus COL1. (A) AZD0530 induced a dose-dependent reduction in D2.0R cell numbers as determined by MTS assay. (B) Target inhibition (pSrc and pFAK) by AZD0530 (250 nM) at 72 hours in D2.0R cells on BME plus COL1. ${ }^{*} P<0.05$, ${ }^{* *} P<0.01$, and ${ }^{* * *} P<0.001$ compared with day 0 of each dose. (C) Reduced Src expression in shRNA clones by Western blot analysis. (D) MTS assay of parental, shNT, and shSrc D2.0R cells (clones 1, 2, 3, and 4) on BME plus COL1. ${ }^{*} P<0.05$ compared with $t=0$ hours of each dose. (E) MTS assay of AZD0530-treated D2.0R cells and (F) shSrc D2.0R cells on BME.

assay, we tail-vein injected tumor cells into mice, with removal of the lungs 15 minutes later and culture of the lung sections in vitro (24). The PuMA assay recapitulated the in vivo increase in metastatic burden in fibrotic lungs compared with that seen in nonfibrotic lungs (Supplemental Figure 2B). Treatment of D2.0R cells in fibrotic lung sections with $250 \mathrm{nM}$ AZD 0530 beginning 5 hours after lung removal completely abrogated the increase in metastatic burden to the levels seen in nonfibrotic lungs $(48,995 \pm 7,230$ versus 43,161 $\pm 19,695$ pixels $^{2}$ ) over the course of 21 days (Supplemental Figure 2B). These results suggest that the PuMA assay is a useful surrogate system for studying the dormant-to-proliferative switch and pharmacological interventions in real time.

SFK inbibition does not revert proliferating metastatic lesions to a dormant state. We next determined whether the inhibition of Src in existing, proliferative metastatic lesions would revert the cells to a dormant state or cause the metastatic lesions to regress in an intervention regimen. D2.0R GFP cells were tail vein-injected into $\mathrm{CD} 1^{n u / n u}$ mice 3 weeks following the intranasal instillation of Ad-empty or Ad-TGF- $\beta 223 / 225$. Twenty-one days after tail-vein injection of the cells and after proliferative lesions had developed in the fibrotic lungs of mice, a group of mice was euthanized to determine baseline lung metastatic burden, and the remaining mice received vehicle or AZD0530 gavage daily. SFK inhibition by AZD0530 in this regimen was strikingly less effective in reducing the established metastatic burden in the fibrotic lungs as compared with the prevention regimen discussed above. In fact, in this regimen, AZD0530 was only able to prevent the further increase in metastatic burden observed between 3 and 6 weeks after tail-vein injection of D2.0R cells in fibrotic lungs (Figure 2E), but did not alter the percentage of dormant cells versus proliferative lesions in both normal and fibrotic lungs (Figure $2 \mathrm{~F}$ ).

SFK inhibition in D2.0R cells on COL1-enriched BME induces a reversible G1/S cell cycle arrest with the upregulation of nuclear 227 . The proliferative index of D2.0R cells on BME or BME plus COL1 was determined as the percentage of cells expressing Ki67. The proliferative index of D2.0R cells on BME was low 24 hours after seeding (Figure 3, A and B) and was not significantly altered by AZD0530, demonstrating that SFK inhibition does not affect quiescent D2.0R cells. After 24 hours on BME plus COL1, however, D2.0R cells exhibited a 6-fold increase in the percentage of Ki67 positivity as compared with D2.0R cells 
A

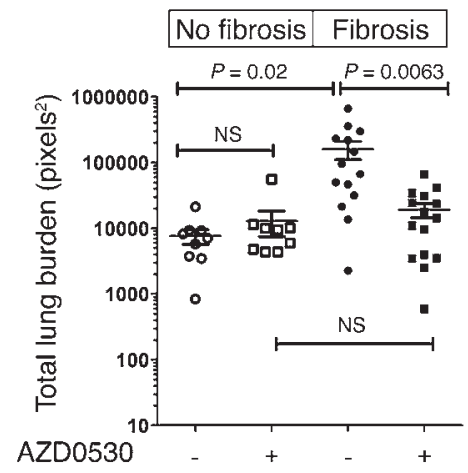

C

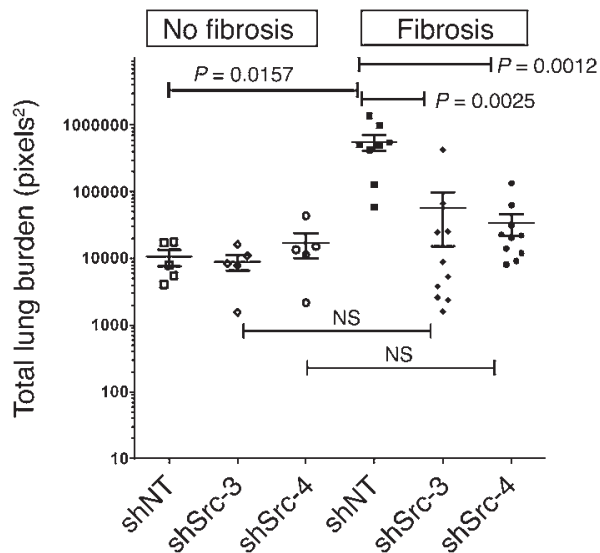

E Ad-empty or IV cell injection

End Ad-TGF- $\beta^{223 / 225}$

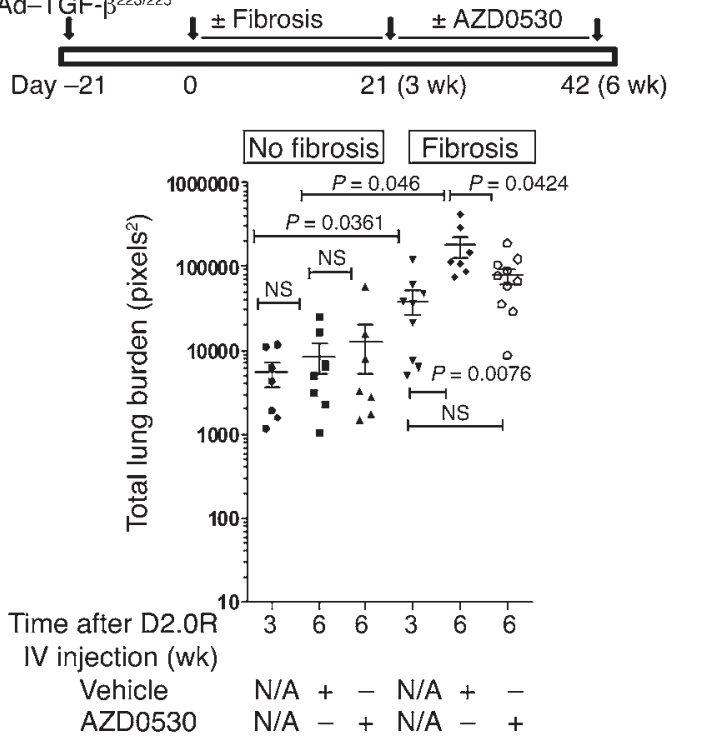

B

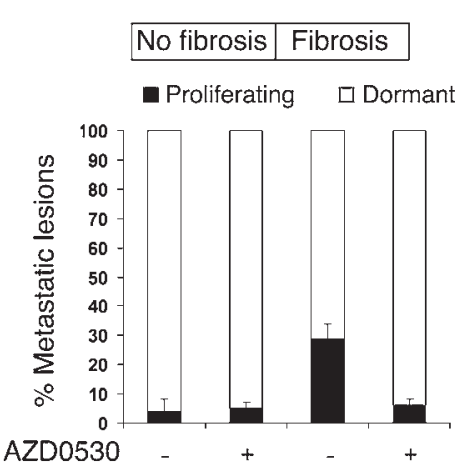

D
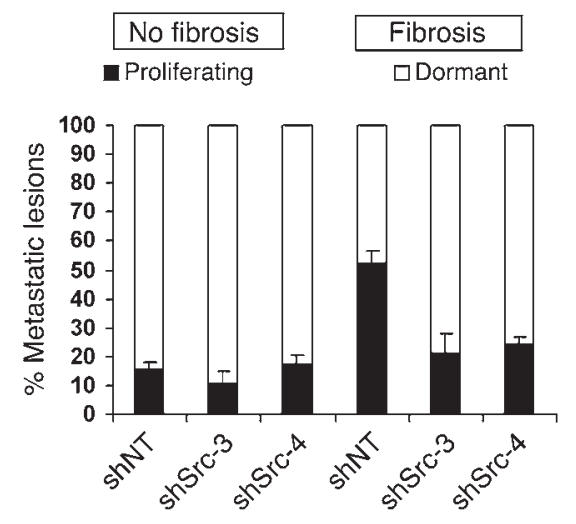

F

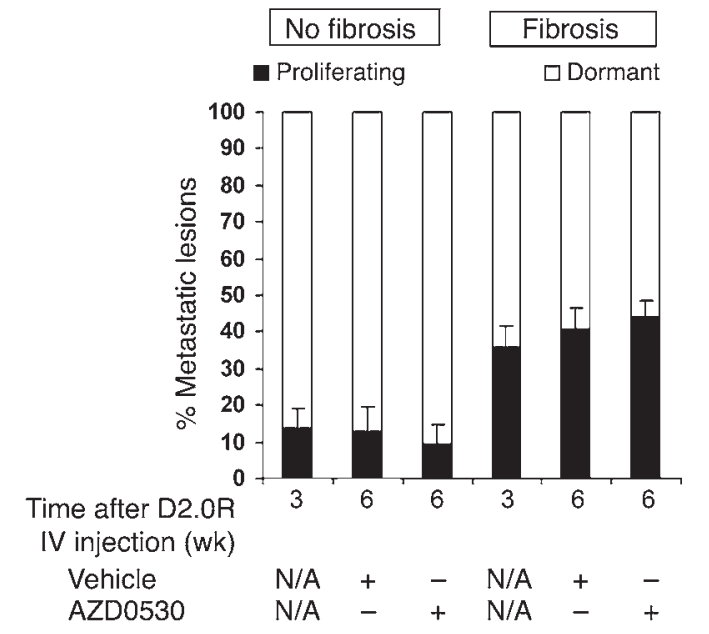

Figure 2

SFK inhibition prevents the dormant-to-proliferative switch of D2.0R cells in fibrotic lungs but does not lead to regression of established lesions. (A) Total lung surface burden of CD1 ${ }^{11 / n u}$ mice receiving Ad-empty (No fibrosis) or Ad-TGF- $\beta^{223 / 225}$ (Fibrosis) and tail-vein injections of $1 \times 10^{6}$ D2.0R GFP cells, followed 24 hours later by $50 \mathrm{mg} / \mathrm{kg}$ body weight of AZD0530 daily for 21 days. (B) Lesions $<1,000$ pixels 2 represent single dormant cells and lesions $>1,000$ pixels 2 represent proliferative lesions. (C) Similar experiment as in $\mathbf{A}$ using $10^{6}$ shNT D2.0R GFP or shSrc D2.0R GFP cells (clones 3 and 4). Lungs were evaluated 21 days after tail-vein injections. (D) Lesion distribution as determined in B. (E and F) Intervention experiment: experiment as in A, except gavage with $50 \mathrm{mg} / \mathrm{kg}$ body weight of AZD0530 was started 21 days after tail-vein injection of cells (schematic provided in E). (F) Lesion distribution determined as in B. No significant difference was detected between groups. 
A

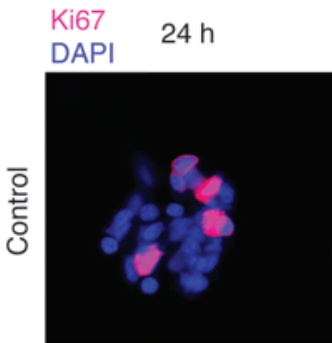

峁
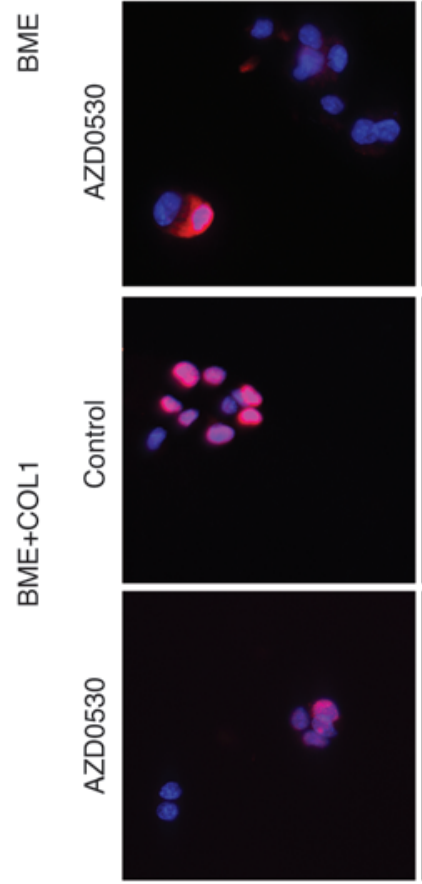

C
$48 \mathrm{~h}$
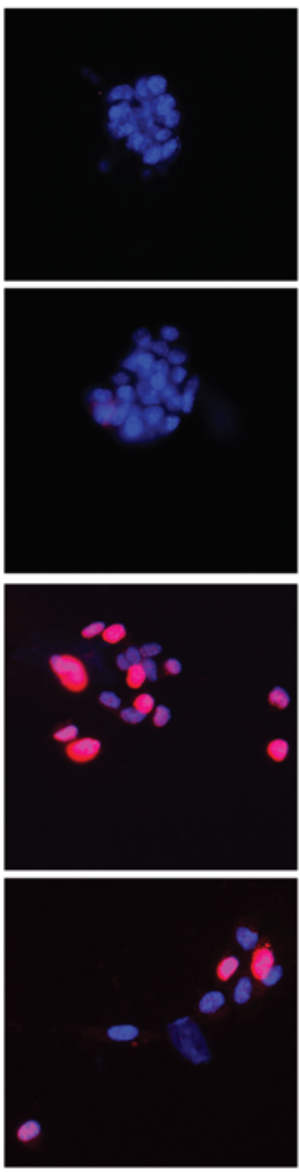
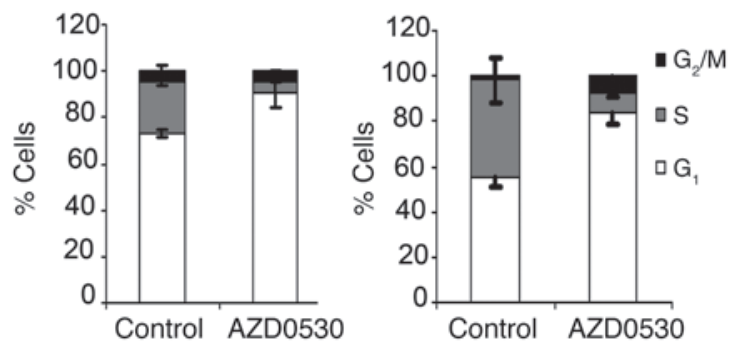

$72 \mathrm{~h}$
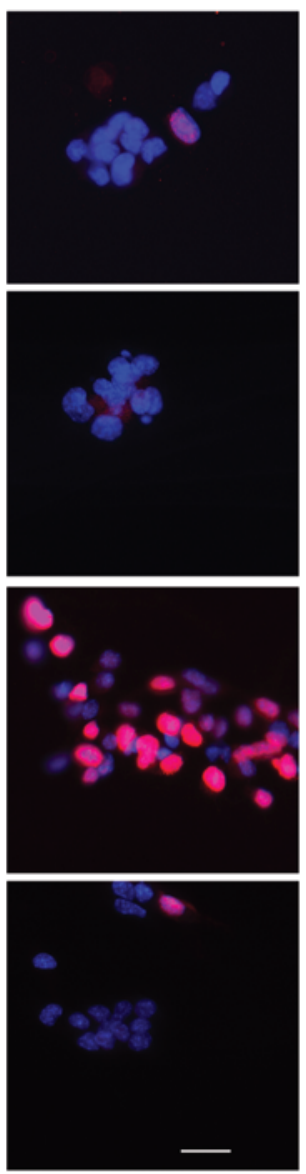
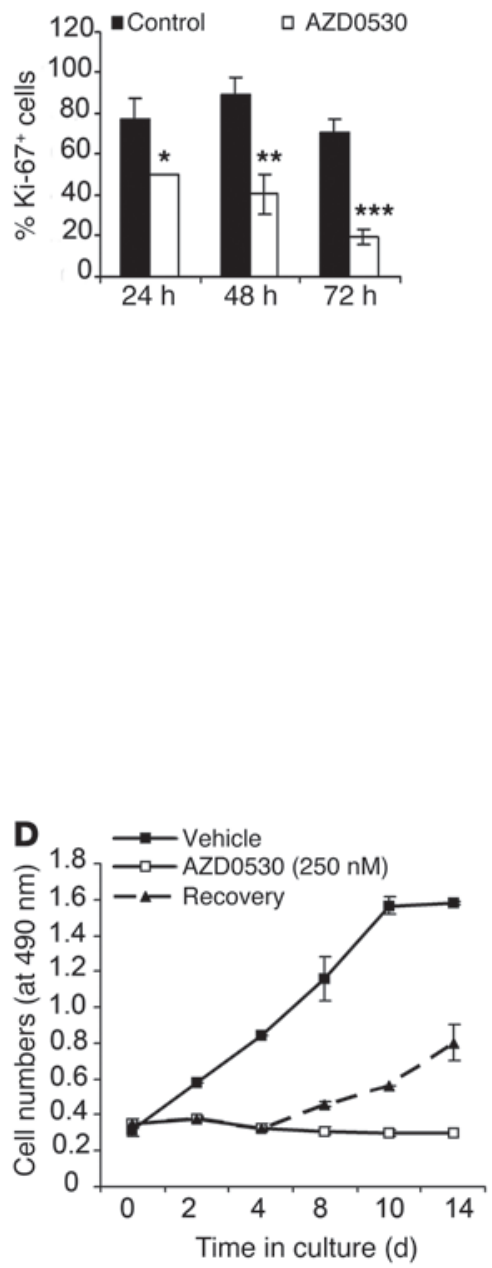

\section{Figure 3}

Effect of AZD0530 on proliferation and cell cycle profile of D2.0R cells on BME or BME plus COL1. (A) Representative Ki67 immunofluorescence of D2.0R cells on BME (upper panels) or BME plus COL1 (lower panels) treated with $250 \mathrm{nM}$ AZD0530 or vehicle (scale bar: 25 um), with (B) quantification. ${ }^{\star} P=0.04 ;{ }^{* *} P=0.0058 ;{ }^{* * \star} P \leq 0.0001$. (C) Cell cycle profile of D2.0R on BME (left) or BME plus COL1 (right) treated with $250 \mathrm{nM}$ AZD0530 or vehicle for 72 hours. (D) MTS assay of D2.0R cells treated with 250 nM AZD0530 or vehicle for 14 days or for 4 days, after which cells were washed and supplemented with vehicle for 10 days (dashed line indicates the recovery phase).

on BME $(70 \pm 10 \%$ versus $12.97 \pm 5.5 \%$, respectively). The proliferative index was significantly reduced by AZD0530 across the 72-hour treatment period (Figure 3, A and B). A G1 phase arrest was demonstrated with $80.5 \% \pm 4.5 \%$ of AZD0530-treated cells accumulating in the $\mathrm{G} 1$ phase versus $52.0 \% \pm 4.0 \%$ of unsynchronized untreated cells (Figure 3C). Importantly, this cell cycle arrest of D2.0R cells on BME plus COL1 was reversible, as withdrawal of AZD0530 after 4 days of treatment resulted in the resumption of proliferation (Figure 3D). p27 expression was reduced at both the mRNA and protein levels (Figure 4A) and localized to the cytoplasm of D2.0R cells seeded on BME plus COL1 compared with cells on BME only (Figure 4B). We observed a reduction in p27 mRNA in D2.0R cells 12 hours after seeding on BME plus COL1 compared with that seen in cells on BME only (Supplemental Figure 3A). Moreover, the pretreatment of D2.0R cells with the proteosomal inhibitor MG132 partially restored p27 levels on BME plus COL1 (Supplemental Figure 3B), 
A

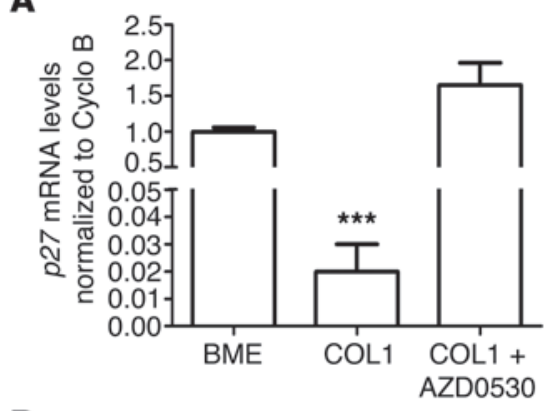

B

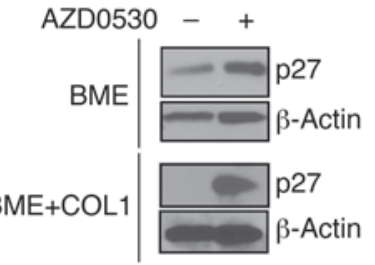

D
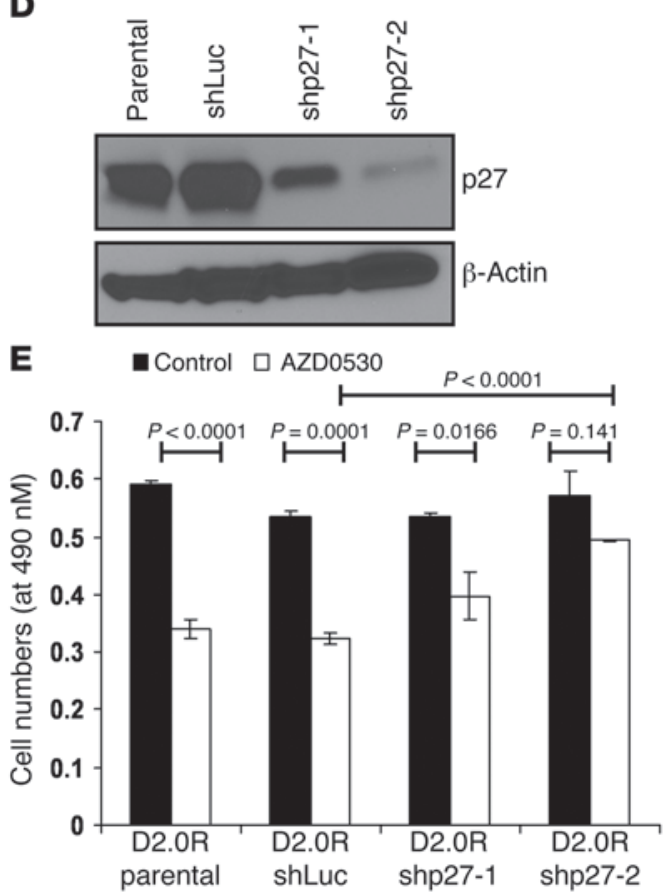

C
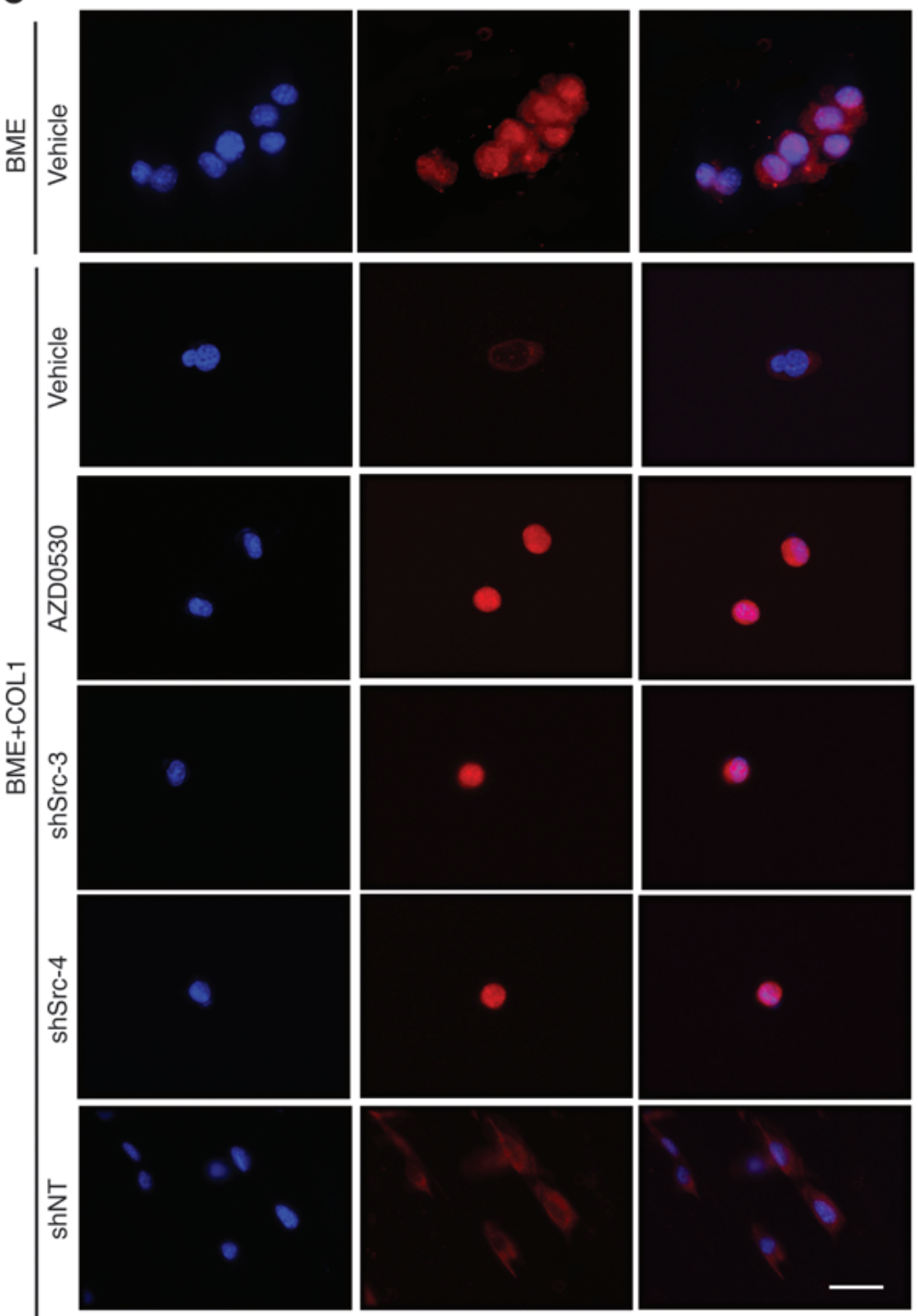

DAPI

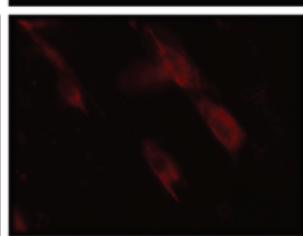

p27

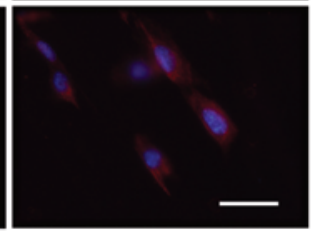

Merge

Figure 4

Src inhibition induces an upregulation of nuclear p27 that is reduced and primarily cytoplasmic in D2.0R cells on BME plus COL1. p27 expression determined by quantitative PCR (A), Western blotting (B), and immunofluorescence staining (C) of D2.0R cells seeded on BME or BME plus COL1 treated with AZD0530 or vehicle, and shNT and shSrc D2.0R cells seeded on BME plus COL1 for 72 hours. Scale bar: $25 \mu \mathrm{m}$. ${ }^{* * *} P=0.001$ compared with BME. (D) p27 levels by Western blot analysis in parental, shLuc, and shp27-1 and shp27-2 D2.0R cells. (E) MTS assay of cells from D on BME plus COL1 treated with 250 nM AZD0530 or vehicle for 72 hours.

suggesting that activation by COL1 exerts both transcriptional and posttranscriptional effects on p27 levels. On the other hand, we observed that pretreatment with AZD0530 resulted in the upregulation of p27 at the mRNA and protein levels (Figure 4, $\mathrm{A}$ and $\mathrm{B})$, which required de novo transcription, since pretreatment with the transcription inhibitor actinomycin $D$ abolished the AZD0530-induced upregulation of p27 mRNA (Supplemental Figure 3C). AZD0530-mediated upregulation of p27 was characterized by the nuclear accumulation of p27 (Figure 4C). We found that Src knockdown in shSrc D2.0R cells (clones 3 and 4) on BME plus
COL1 also resulted in strong nuclear accumulation of $\mathrm{p} 27$, while control shNT D2.0R cells exhibited a markedly reduced expression of p27 that was exclusively cytoplasmic (Figure 4C). We have previously demonstrated that D2.0R cells with knockdown of integrin $\beta 1$ remain quiescent in BME plus COL1 (11); we now show that these cells also exhibit strong nuclear p27 accumulation (Supplemental Figure 3D). The induction of nuclear p27 in the absence of Src activation appears to represent a major feature of the dormant cells.

To determine the role of $\mathrm{p} 27$ in preventing the dormant-to-proliferative switch of AZD0530-treated cells, we established two 
A

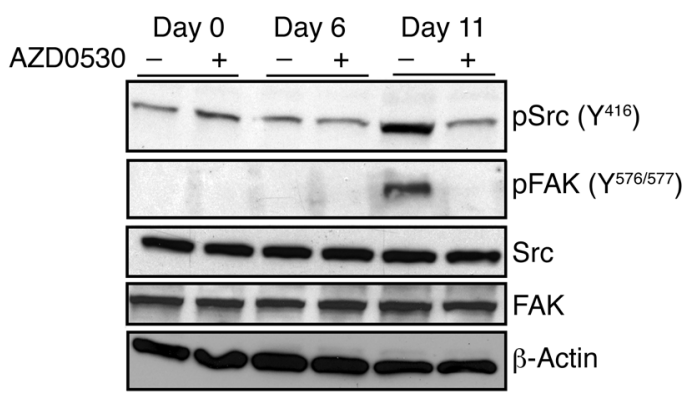

\section{B}

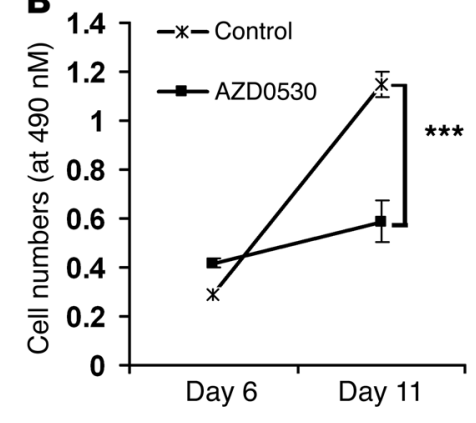

C

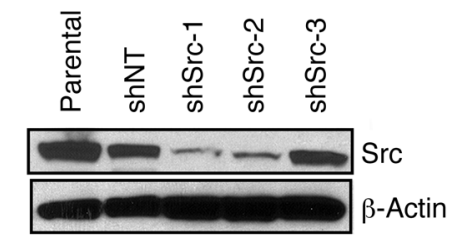

D

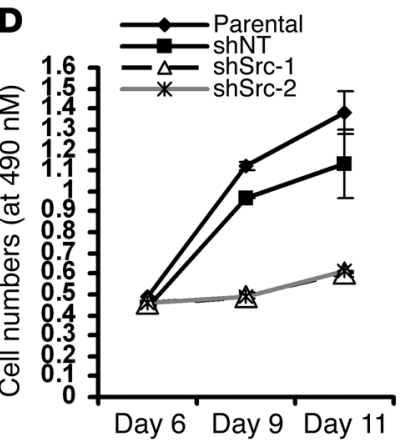

E

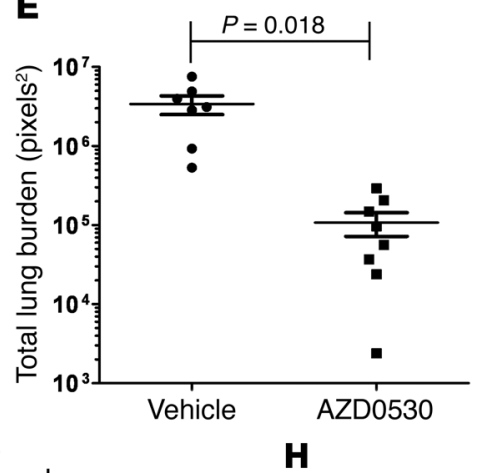

H
F
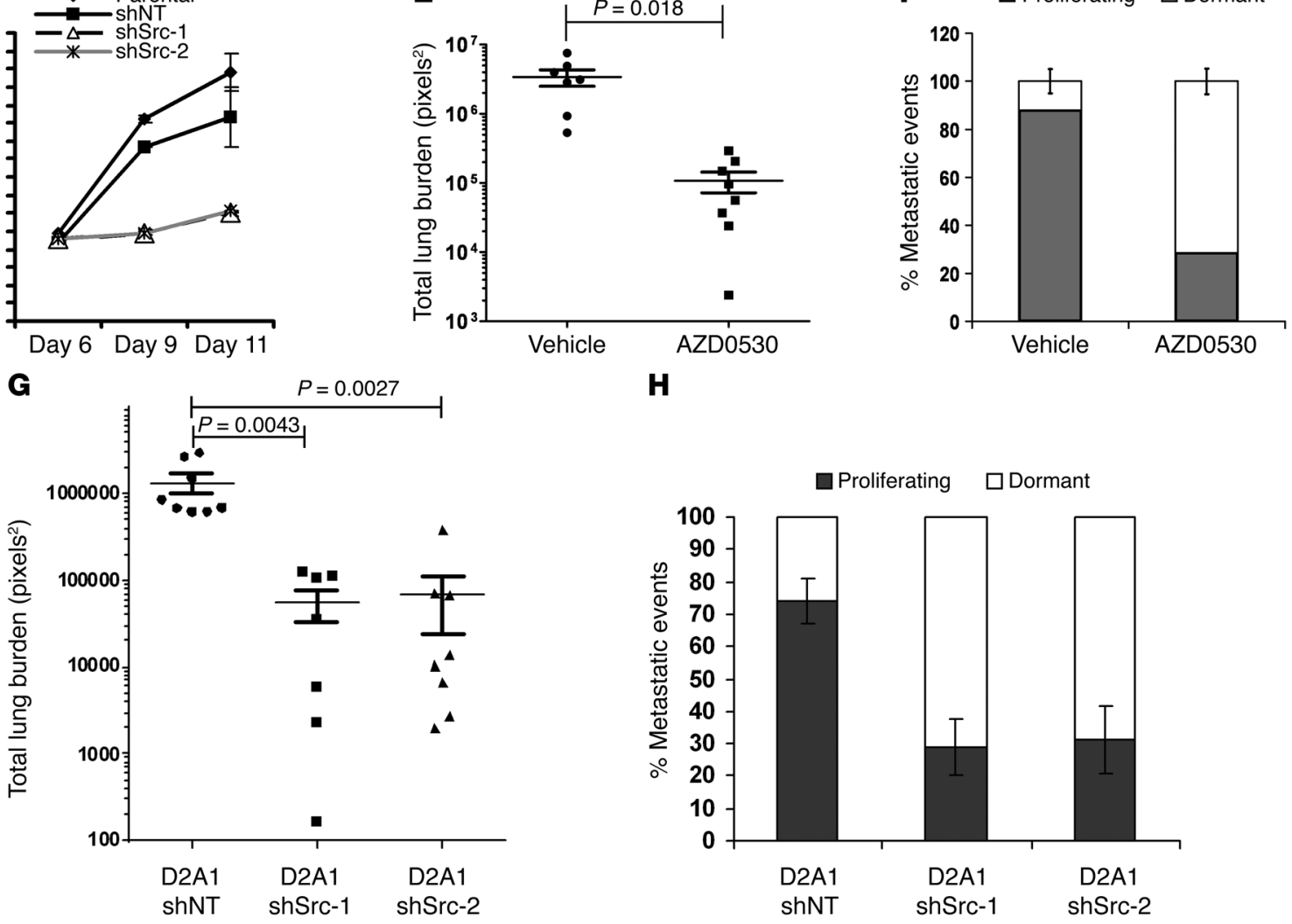

Figure 5

SFK inhibition prevents the spontaneous outbreak of highly metastatic D2A1 cells. (A) Representative Western blots of pSrc ( $\left.\mathrm{Y}^{416}\right)$, pFAK (Y576/577), total Src, and total FAK on days 0, 6, and 11 of D2A1 on BME with or without AZD0530 (250 nM). (B) MTS assay of D2A1 cells seeded on BME and treated with or without $250 \mathrm{nM}$ AZD0530 for 11 days. (C) Western blot of D2A1 shSrc clones and cell numbers by MTS assay (D) after 11 days on BME. ${ }^{* *} P<0.0001$. (E) Total lung surface metastatic burden and $(\mathbf{F})$ distribution of $<1,000$ and $>1,000$ pixels $^{2}$ lesions representing dormant and proliferating cells, respectively in CD1 ${ }^{\text {nu/nu }}$ mice injected with D2A1 GFP cells and gavaged 24 hours later with $50 \mathrm{mg} / \mathrm{kg}$ body weight of AZD0530 for 21 days. (G) Total lung surface metastatic burden and (H) distribution of $<1,000$ and $>1,000$ pixels ${ }^{2}$ lesions representing dormant and proliferating cells, respectively, in CD1 ${ }^{\text {nu/nu }}$ mice injected with D2A1 GFP stably expressing shNT or shSrc (clones 1 and 2).

clonal D2.0R cell lines stably expressing p27 shRNA, with a resultant $40 \%$ and $80 \%$ reduction in p27 protein levels, respectively (Figure 4D), while a luciferase shRNA-expressing D2.0R cell line served as a control. Whereas AZD0530 treatment resulted in the expected approximately $40 \%$ reduction in the number of parental and luciferase shRNA cells seeded on BME plus COL1, p27 shRNA cell numbers were decreased by only $25 \%$ and $13 \%$ in AZD0530treated D2.0R shp27-1 and shp27-2 clones, respectively (Figure 4E). On the other hand, we found that knockdown of p27 in D2.0R cells seeded on BME had no effect on cell numbers. Therefore, knockdown of p27 mitigates the inhibitory effect of Src inhibition of the dormant-to-proliferative switch and reduces by 3 -fold the inhibitory effect of AZD0530 on proliferation.

SFK inhibition prevents the spontaneous dormant-to-proliferative switch of mouse D2A1 and human BC cell lines in $3 D$ culture and in vivo with concomitant upregulation of $p 27$. We next examined whether SFK inhibition can also modulate the spontaneous proliferative outbreak of the metastatic D2A1 mammary tumor cells. D2A1 cells have been shown by our lab and others to remain dormant for approximately 6 days on BME or for 1 to 3 weeks in normal lung tissue, 
A
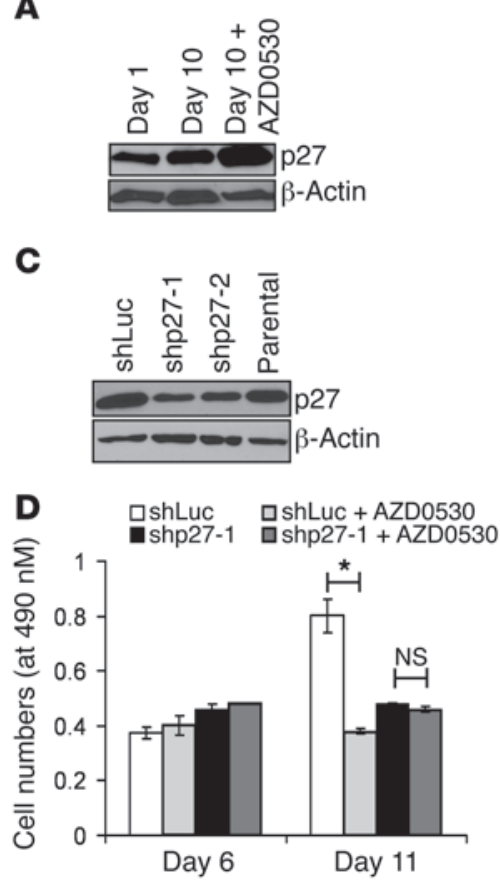

B
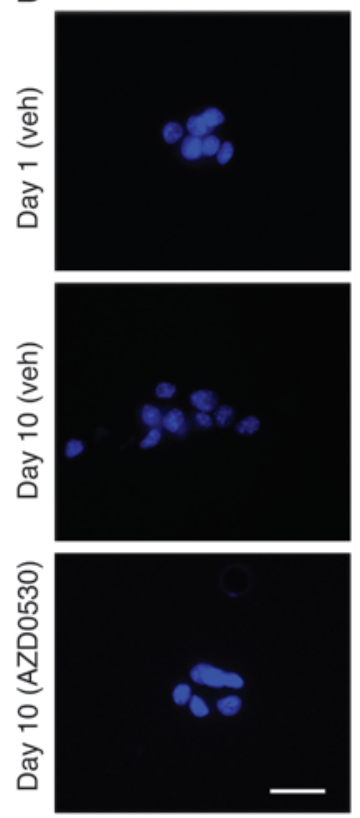

DAPI
D2A1 on BME
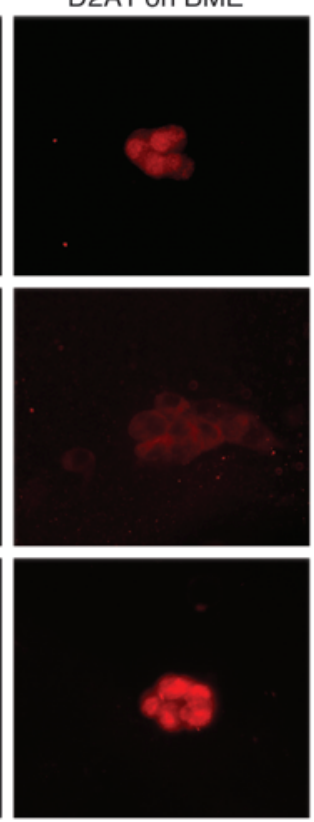

p27
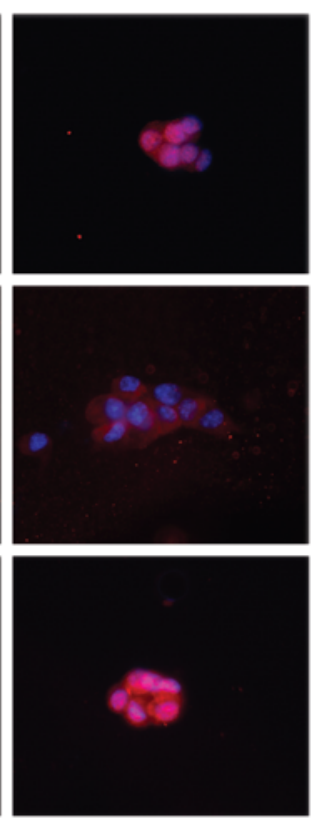

Merge

Figure 6

AZD0530 mediated inhibition of the spontaneous proliferative switch is associated with nuclear accumulation and upregulation of p27. (A) Western blot and (B) immunofluorescence of p27 levels on days 1 and 10 in D2A1 cells seeded on BME and treated with 250 nM AZD0530 or vehicle. Scale bar: $25 \mu \mathrm{m}$. (C) Reduction in p27 levels by shRNA shown by Western blot, and (D) MTS assay values of shLuc and shp27 D2A1 cells. ${ }^{*} P=0.0253$.

after which they begin to spontaneously proliferate (10). Proliferation of D2A1 cells has been shown to occur concomitantly with an increase in COL1 expression in lung tissue (11). D2A1 cells were seeded on BME and treated with AZD0530 or vehicle for 11 days. Whereas AZD0530 had no effect on D2A1 cell numbers on day 6 of treatment (pre-spontaneous outbreak), it prevented the 4-fold increase in cell numbers we observed in untreated D2A1 cells on day 11 after the spontaneous proliferative outbreak had occurred (Figure 5B). Interestingly, the spontaneous proliferation of D2A1 was accompanied by an increase in Src activity as evidenced by a 4-fold increase in $\mathrm{pSrc}\left(\mathrm{Y}^{416}\right)$ and significant phosphorylation of FAK $\left(\mathrm{Y}^{576 / 577}\right)$ (Figure 5A). On the other hand, AZD0530-treated D2A1 cells exhibited a marked reduction in $\mathrm{pSrc}\left(\mathrm{Y}^{416}\right)$ and $\mathrm{pFAK}$ $\left(\mathrm{Y}^{576 / 577}\right)$, demonstrating reduced Src activity (Figure 5A). We observed similar results with the two clones stably expressing shSrc (shSrc-1 and -2, with 76\% and 74\% Src knockdown, respectively; Figure 5C) that failed to spontaneously proliferate on BME, whereas parental and shNT D2A1 cells underwent a spontaneous proliferative outbreak (Figure 5D). Human BC MDA-MB-231 cells have been previously shown to undergo a similar spontaneous proliferative outbreak after 3 days in 3D culture (10). We similarly demonstrate that treatment with AZD0530 prevented this proliferative switch in MDA-MB-231, whereas untreated cells that proliferated exhibited a 6-fold increase in Src activity as determined by increased pSrc $\left(\mathrm{Y}^{416}\right)$ expression, with no effect on total Src levels (Supplemental Figure 4, B and C).

In order to determine the efficacy of SFK inhibition on the spontaneous outbreak of D2A1 cells in vivo, CD $1^{n u / n u}$ female mice were tail-vein injected with $1 \times 10^{6}$ D2A1 GFP cells and treated with AZD0530 (50 mg/kg body weight) or vehicle by gavage 24 hours later for 21 days in a prevention regimen. The metastatic lung burden in vehicle-gavaged mice was measured as $5 \times 10^{6}$ pixels $^{2}$ per mouse, whereas mice receiving AZD0530 for 21 days demonstrated a 50-fold reduction of metastatic burden in the lungs, with approximately $1 \times 10^{5}$ pixels ${ }^{2}$ per mouse (Figure $5 \mathrm{E}$ ). Importantly, the distribution of dormant versus proliferative lesions was markedly altered by AZD0530, with the dormant population increasing from $11.93 \% \pm 5.05 \%$ in vehicle-gavaged mice to $71.45 \% \pm 5.36 \%$ of the total lung lesions in the AZD0530-treated mice (Figure 5F).

To further demonstrate the critical role of Src, specifically, in the spontaneous dormant-to-proliferative switch of D2A1 cells in normal lungs, we performed in vivo experiments using shNT or shSrc D2A1 cells (clones 1 and 2). Twenty-one days after tail-vein injection, we found that shNT D2A1 cells resulted in an average metastatic burden of $1.32 \times 10^{6}$ pixels $^{2}$ per mouse, whereas clones 1 and 2 of the shSrc of D2A1 cells resulted in a 24- and 20-fold reduction, respectively, in the average lung metastatic burden compared with that seen in shNT cells (Figure 5G). Importantly, the knockdown of Src in D2A1 cells resulted in a significant increase in the percentage of dormant lesions $(71.25 \% \pm 8.56 \%$ and $68.83 \% \pm 10.65 \%$ for $\operatorname{shSrc}$ clones 1 and 2 compared with only $26.11 \% \pm 6.79 \%$ in shNT D2A1 cells) (Figure $5 \mathrm{H}$ ), similar to the effects of AZD0530.

Interestingly, our examination of p27 levels and its subcellular distribution in D2A1 cells pre- and post-spontaneous outbreak revealed that $\mathrm{p} 27$ levels are not reduced upon the spontaneous outbreak of D2A1 cells. p27 relocalized to the cytoplasm in D2A1 cells that underwent the spontaneous dormant-to-proliferative switch, as previously reported (10), but not in shSrc D2A1 cells or shITGB1 D2A1 cells that lack Src activation and are unable to spontaneously proliferate (Supplemental Figure 4A). Importantly, 
A

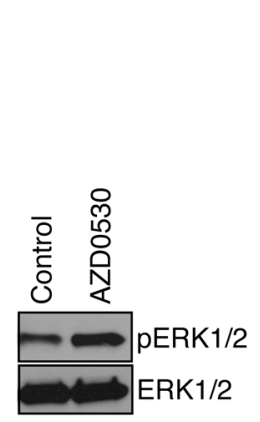

D

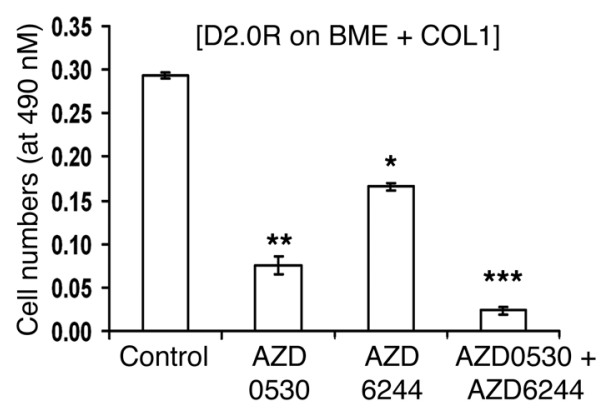

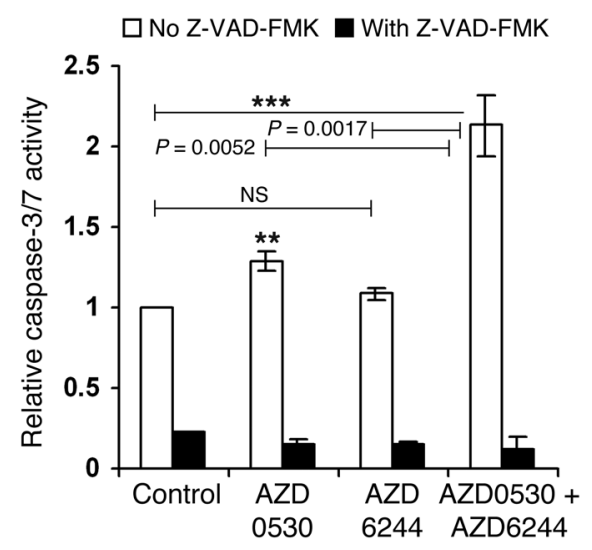

E
C
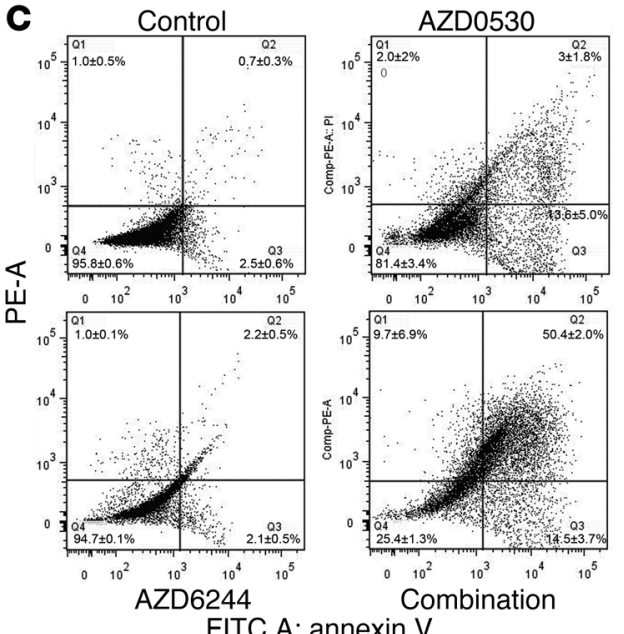

FITC A: annexin V

F

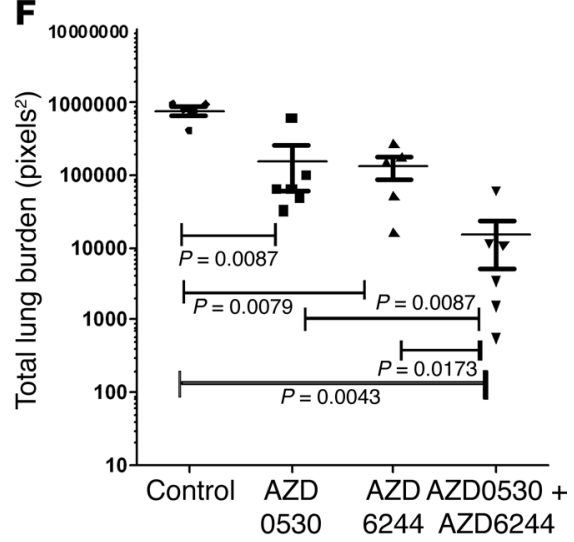

\section{Figure 7}

Combination of AZD0530 and AZD6244 induces apoptosis of D2.0R cells on BME plus COL1 and dramatically reduces their metastatic burden in fibrotic lungs. (A) pERK1/2 levels in D2.0R cells treated with $250 \mathrm{nM}$ AZD0530 or vehicle for 72 hours. (B) Caspase 3/7 assays of D2.0R cells treated with AZD0530 (250 nM), AZD6244 (500 nM), or both with or without Z-VAD-FMK for 48 hours. ${ }^{* \star} P=0.0034 ;{ }^{* \star \star} P=0.0011$. (C) Annexin V/PI staining of D2.0R cells treated with vehicle, AZD0530, AZD6244, or their combination, as above, for 48 hours with quantification \pm SEM from three experiments. (D) MTS assay for D2.0R seeded on BME plus COL1 and treated, as above, for 96 hours. ${ }^{*} P=0.0051$, ${ }^{* \star} P=0.0029$, and ${ }^{* \star *} P=0.0007$ compared with vehicle-treated cells; also, $P=0.0061, P=0.0194$, and $P=0.0025$ comparing AZD0530 versus AZD6244, AZD0530 versus combination, and AD6244 versus combination, respectively. (E) MTS values of similarly treated D2.0R cells with or without Z-VAD-FMK at 48 hours. ${ }^{\star} P=0.0282,{ }^{* \star} P=0.0016$, and ${ }^{\star \star \star} P=0.0013$ compared with control vehicle-treated D2.0R cells. (F) Lung metastatic burden represented in pixels ${ }^{2}$ of CD1 1nu/nu mice instilled with Ad-TGF- $\beta^{223 / 225}$, then tail-vein injected with $1 \times 10^{6} \mathrm{D} 2.0 \mathrm{R}$ GFP cells, followed by gavage 24 hours later with vehicle, AZD0530, AZD6244, or their combination once daily for 21 days.

AZD0530 treatment of D2A1 cells for 10 days resulted in both the upregulation of $\mathrm{p} 27$ and its relocalization to the nucleus (Figure 6, $A$ and B). We also observed p27 nuclear relocalization upon SFK inhibition in AZD0530-treated human MDA-MB-231 cells in the absence of an increase in p27 protein levels (Supplemental Figure 4D). This raises the intriguing possibility that upregulation of cytoplasmic p27 may contribute to the proliferative outbreak of D2A1 and MDA-MB-231 cells.

To further examine the role of p27 in the inhibition of the spontaneous outbreak of D2A1 cells, D2A1 cells stably expressing p27 shRNA were established with a 60\% (shp27-1) and 50\% (shp27-2) reduction in p27 protein levels (Figure 6C). shLuc D2A1 cells underwent a spontaneous proliferative outbreak on BME by day 11 in culture (as did the D2A1 parental cells), which was prevented in both cases by AZD0530 treatment (Figure 6D). shp27 D2A1 cells failed to outbreak on BME by day 11, and AZD0530 treatment did not reduce cell numbers (Figure 6D). This further supports a role for cytoplasmic p27 in contributing to the proliferation of metastatic D2A1 cells, which could be prevented by AZD0530 treatment and the nuclear retention of p27. Importantly, we found that the forced expression of cytoplasmic, but not wild-type, p27 can substantially increase the proliferation of highly metastatic D2A1 and poorly metastatic D2.0R cells on BME plus COL1 matrices (Supplemental Figure 5).

Targeting MEK and SFK in combination reduces the viability and number of dormant cells. Since earlier studies in other model systems have demonstrated that the ERK1/2 to p38 MAPK ratio is critical in regulating quiescence (low ratio) or proliferation (high ratio) of dormant cancer cells (25), we examined the effect of Src inhibition on the activation p38 MAPK. AZD0530 treatment of D2.0R cells seeded on BME plus COL1 did not result in a reduction in p38 MAPK activity, as evidenced by a lack of change in the phos- 
phorylation of $\mathrm{T}^{180} / \mathrm{Y}^{182}$ of p38 MAPK (Supplemental Figure $6 \mathrm{~A}$ ). Interestingly, we found that two p38 MAPK inhibitors (SB202190 and SB203580) resulted in increased proliferation of D2.0R cells on BME plus COL1 as compared with untreated D2.0R cells. This suggests that $\mathrm{p} 38 \mathrm{MAPK}$ exhibits an inhibitory effect on D2.0R proliferation that is not completely relieved by the addition of COL1. The proliferative effect of p38 MAPK inhibition was completely abrogated by cotreatment with AZD0530 (Supplemental Figure 6B).

We have previously shown that the COL1-induced outbreak of D2.0R cells is accompanied by activation of the ERK/MAPK pathway (11). We have now extended this observation by showing that ERK1/2 activation also occurs with the spontaneous proliferative outbreak of metastatic D2A1 and MDA-MB-231 cells cultured on BME (Supplemental Figure 7A and Supplemental Figure 10), concomitant with the activation of Src (Figure 5A and Supplemental Figure $4 \mathrm{~A})$. This suggests that the inhibition of ERK1/2 activation is an additional target in preventing the dormant-to-proliferative switch. We observed that the inhibition of MEK1/2 (upstream activators of MAPK) using the inhibitors U0-126 or AZD 6244 reduced the proliferation of D2.0R cells seeded on BME plus COL1 (Supplemental Figure 7B) and inhibited the spontaneous proliferation of D2A1 and MDA-MB-231 cells (Supplemental Figure 7, C and D). However, SFK inhibition using AZD0530 did not result in the inactivation of ERK1/2. ERK1/2 phosphorylation is maintained in AZD0530-treated D2.0R cells on BME plus COL1 (Figure 7A) as well as in AZD0530-treated D2A1 and MDA-MB-231 cells cultured on BME after 8 and 10 days, respectively (Supplemental Figure 7E and Supplemental Figure 10). These results suggest that the combination of SFK and MEK1/2 inhibitors might have a more potent effect in preventing the dormant-to-proliferative switch.

To elucidate the effect of combining SFK and MEK1/2 inhibitors on the dormant-to-proliferative switch and survival of cells, D2.0R cells were seeded on BME plus COL1 and treated with either $250 \mathrm{nM}$ AZD0530, $500 \mathrm{nM}$ of the MEK1/2 inhibitor AZD6244, or both. Although single-drug treatment with AZD0530 only resulted in a modest increase in caspase $3 / 7$ activity 48 hours after treatment, combining AZD0530 and AZD6244 resulted in a significantly higher caspase $3 / 7$ activity than each inhibitor alone (Figure 7B), which we did not observe using AZD0530 and p38 MAPK inhibitors (Supplemental Figure 6C). The combination of AZD0530 and AZD6244 resulted in an approximately 50-fold increase in the population of propidium iodide-positive (PI-positive) and annexin V-positive late apoptotic D2.0R cells compared with that of either inhibitor alone (Figure 7C), and combining AZD0530 and AZD6244 resulted in a further decrease in cell numbers compared with that seen with either inhibitor alone after 2 days of treatment (Figure 7D). Cotreatment of AZD0530 and AZD6244 with the pan-caspase inhibitor Z-VAD-FMK prevented the increase in apoptosis of D2.0R cells and resulted in the restoration of cell numbers to those observed with AZD0530 alone (Figure 7, B and E). Therefore, the addition of the MEK1/2 inhibitor to AZD0530 reduces the viability of dormant cells on BME plus COL1. We found that the same combination therapy had a minimal effect on normal mammary epithelial cells (Supplemental Figure 8A). We evaluated the reversibility of this combination treatment after 3 days of treatment. Whereas D2.0R cells treated with either drug alone showed enhanced proliferation on day 5 after drug withdrawal, we observed that D2.0R cells treated with AZD0530 plus AZD6244 failed to proliferate after 8 days in culture (5 days after drug withdrawal) (Supplemental Figure 8B).
This combination treatment was also evaluated in vivo. We tailvein injected the mice induced to develop lung fibrosis with D2.0R cells, as previously described, and gavaged them 24 hours later with AZD0530 $(50 \mathrm{mg} / \mathrm{kg})$ or AZD6244 (25 mg/kg). AZD0530 and AZD6244 resulted in an approximately 5 -fold reduction in the average total lung burden compared with vehicle-treated mice. However, the combination therapy (which did not result in any weight loss compared with that seen with the single inhibitors or vehicle) (Supplemental Figure 8C), resulted in a statistically significant 53-fold reduction compared with vehicle-treated mice $(P=0.0043)$ (Figure 7F). The superior efficacy of the combination treatment was also recapitulated in the ex vivo lung metastasis assay (Supplemental Figure 8D).

Importantly, we also observed the increase in caspase activation upon cotreatment with MEK1/2 and Src inhibitor in the COL1-induced or spontaneous dormant-to-proliferative switch of other cancer cells tested. Human MDA-MB-231 cells were induced to proliferate on BME plus COL1 (Supplemental Figure 9A), and treatment with both inhibitors resulted in increased caspase 3/7 activation compared with either inhibitor alone (Supplemental Figure 9B). Similarly, the osteosarcoma cell line K7M2, which remains quiescent for 3 days on BME (10) and proliferates within 24 hours on BME plus COL1, exhibited increased caspase 3/7 activity with the combination of AZD0530 plus AZD6244 compared with that observed with the individual inhibitors (Supplemental Figure 9, C and D). This enhanced apoptotic effect of the combination treatment was also observed in the spontaneous outbreak of other cancer cell lines, including K7M2 and the highly metastatic 4T1 BC cells upon their spontaneous proliferative outbreak on days 4 and 2, respectively (Supplemental Figure 9, E-G).

Taken together, this demonstrates that the combination of SFK and MEK $1 / 2$ inhibitors has superior efficacy in inhibiting the dormant-to-proliferative switch of cancer cells, reducing the burden of dormant $\mathrm{BC}$ cells and decreasing the metastatic burden in vivo.

\section{Discussion}

Disease relapse from dormant tumor cells after years of latency is a major cause of mortality (26). In this study, we have identified a combination therapy using SFK and MEK1/2 inhibitors that reduces the viability of dormant cells that are triggered to undergo a dormant-to-proliferative switch.

We have previously shown that a collagen- or fibronectin-rich microenvironment can mediate the switch of BC cells from dormancy to overtly proliferating metastatic lesions in the lung $(10,11)$. This effect is mediated through an ITGB1-dependent pathway that requires Src activation beyond a threshold observed in dormant cells as well as ERK1/2 activation.

We have demonstrated that inhibiting SFK activity with AZD0530 can prevent the proliferative outbreak of dormant cells in a COL1enriched microenvironment using a 3D culture model as well as in a COL1-enriched fibrotic lung environment in vivo. Further, the spontaneous proliferative outbreak, after a short latency period, of the highly metastatic D2A1 and MDA-MB-231 cell lines, which is accompanied by Src activation, is also prevented by the SFK inhibitor.

Although SFK inhibition prevents the COL1-induced proliferative outbreak of dormant cells in $3 \mathrm{D}$ culture and in the fibrotic lung environment, discontinuation of the SFK inhibitor in the presence of COL1 resulted in the proliferation of the dormant cells. SFK inhibition was also not effective in causing the regression of established metastatic lesions in vivo to their baseline level 
prior to AZD0530 administration or in reverting cells back to a dormant state. This result suggests that Src is critical in initiating the proliferation of the dormant cells, but that other factors that are Src independent are used to sustain survival. This observation is consistent with the negligible efficacy of SFK inhibitors in patients with advanced metastatic disease (27). In fact, elevated Src activity is associated with decreased disease-free survival, increased relapse, and lower recurrence-free survival in patients with triple-negative and estrogen receptor-positive BC, underscoring the potential importance of targeting Src (28-30). However, clinical trials with AZD0530 and other dual SFK/ABL inhibitors have shown very modest efficacy in treating advanced triple-negative BCs (31), suggesting that these regimens may miss a "window of opportunity" as the metastatic proliferative switch occurs.

Concomitant with Src activation is the cytoplasmic relocalization of the cell cycle inhibitor p27, as well as a reduction in p27 expression in D2.0R cells when cultured with COL1. Inhibition of SFK by AZD0530 prevented the cytoplasmic relocalization of $\mathrm{p} 27$ in all cell lines tested and induced the increased expression of $\mathrm{p} 27$ in D2.0R and D2A1 cells. Interestingly, increased p27 expression has been recently reported in locoregionally disseminated dormant $\mathrm{BC}$ cells (32). We demonstrated that reduced expression and cytoplasmic localization of $\mathrm{p} 27$ are dependent on Src activation downstream of ITGB1, since cells expressing shSrc or shITGB1 cultured on BME plus COL1 exhibit high levels of nuclear p27 expression. Interestingly, Src activation has been associated with reduced nuclear p27 expression in human BC samples (33), and several Src inhibitors have been shown alone or in combination with other inhibitors to upregulate p27 expression in breast (34) and ovarian (35) cancer cell lines, among others. However, in our dormancy model, p27 induction did not result in as substantial an apoptotic death as reported for ovarian cancer cell lines cultured on plastic (35). Additionally, we have shown that the AZD0530-induced nuclear localization of $\mathrm{p} 27$ is an important mediator of AZD0530 inhibition in spontaneously proliferating, highly metastatic cell lines.

FAK, a mediator of integrin signaling that associates with Src, has also been shown to control p27 levels by a yet-to-be-determined mechanism (36). AZD0530 results in a reduction in phosphorylation of FAK $\left(\mathrm{Y}^{576 / 577}\right)$ through the inhibition of SFK, suggesting that reduced FAK activity (37) could also contribute to the upregulation of p27. Knockdown of p27 in D2.0R cells on BME plus COL1 only partially reduced the effect of SFK inhibition, suggesting that other SFK-dependent signaling mechanisms regulate proliferation of COL1-stimulated D2.0R cells.

Interestingly, we have shown that the spontaneous proliferation of highly metastatic D2A1 and MDA-MB-231 cells is accompanied only by the cytoplasmic relocalization of $\mathrm{p} 27$, but not by a reduction in $\mathrm{p} 27$ protein expression. Importantly, we demonstrated that these highly metastatic cell lines require elevated cytoplasmic p27 levels to undergo spontaneous outbreak, since depletion of p27 in D2A1 cells inhibits their spontaneous proliferation. This suggests that therapeutic strategies that induce the nuclear localization of p27 may prevent the dormant-to-proliferative switch of metastatic cells. Cytoplasmic p27 has previously been shown to be necessary for cancer cell motility of hepatic and BC cells (38-40). Furthermore, epithelial-to-mesenchymal transition (EMT) is known to be associated with a highly motile phenotype $(41,42)$, suggesting that cytoplasmic p27 maybe be involved in this process.

Since inhibition of the dormant-to-proliferative switch is reversible upon withdrawal of the SFK inhibitor and continual treatment is not feasible due to potential side effects (27), additional strategies are required. The ratio of ERK1/2 to p38 MAPK activity has been shown to be a determinant of the proliferative or dormant state of human head and neck cancer cells (25). Similarly, we show that p38 MAPK is inhibitory in our dormancy model, whereas inhibiting p38 MAPK markedly increases the proliferative potential of D2.0R cells primed to proliferate in 3D by the addition of COL1. Since we observed that phosphorylation of ERK1/2 and P38 MAPK is not affected by SFK inhibition of D2.0R cells, we explored whether inhibition of ERK $1 / 2$ by blocking the activation of its upstream activators MEK1/2 could serve as an additional target to prevent the dormant-to-proliferative switch. Importantly, we have shown that using AZD0530 in combination with a MEK1/2 inhibitor (but not a p38 MAPK inhibitor) results in substantial apoptosis and a reduction of D2.0R cell numbers, which are not seen with Src inhibition alone. The superior efficacy of this combination in a prevention regimen was also observed in several other cancer cell lines that are induced to proliferate on COL1 or that are known to spontaneously proliferate on BME. This suggests that ERK1/2 signaling is necessary for the viability of SFK-inhibited cells induced to undergo the dormant-to-proliferative switch by COL1 or that are spontaneously undergoing the proliferative switch. Interestingly, withdrawal of the combination therapy delays and reduces the proliferative outbreak of the dormant D2.0R cells, which is not observed with either inhibitor alone. This demonstrates that the combination treatment is an improved strategy to reduce dormant cells and the dormant-to-proliferative switch. This combination has been shown to inhibit the growth and invasiveness of melanoma cells in 3D cultures (43).

In summary, we have shown that SFK inhibition prevents the COL1-induced proliferative switch of metastatic dormant BC cells and highly metastatic mouse and human BC cells, concomitant with a strong nuclear accumulation of the cell cycle inhibitor p27. The combination of SFK and MEK1/2 inhibition leads to a significant apoptotic response in the dormant population of $\mathrm{BC}$ cells induced to proliferate through changes in the microenvironment or in quiescent cells spontaneously transitioning to a proliferative state. This approach may be of clinical value for reducing tumor recurrence.

\section{Methods}

\section{Cell lines, stable shRNA cell lines, and inbibitors}

Mouse mammary cancer D2.0R (dormant) and D2A1 (metastatic) cells derived from a spontaneous hyperplastic alveolar nodule have been previously described $(9,44,45)$ and were provided by Ann Chambers (London Cancer Center, London, Ontario, Canada). 4T1 cells, obtained from Fred Miller (Barbara Ann Karmanos Cancer Institute, Detroit, Michigan, USA) (46); human MDA-MB-231 cells (ATCC); and mouse osteosarcoma K7M2 cells $(47)$ were grown in $2 \mathrm{D}$ and $3 \mathrm{D}$ culture as previously described $(10,11)$. D2.0R and D2A1 cells were generated to express GFP lentivirally via pSICO and were provided by Tyler Jacks (Massachusetts Institute of Technology, Cambridge, Massachusetts, USA). HEK-293 (ATCC) and Jurkat cells (gift from Noemi Kedei, NCI) were used as positive controls for SFKs. The Src inhibitors used were: PP1 (Enzo Life Sciences) and AZD0530 (AstraZeneca); the MEK1/2 inhibitors used were: AZD6244 (Selleckchem) and U0126 (EMD Millipore). p38 MAPK inhibitors SB202190 and SB203580, actinomycin D, and MG132 were obtained from Sigma-Aldrich.

Wild-type p27 and p27 $\Delta$ NLS-mutant constructs (provided by Giuseppe Viglietto, University of Catanzaro, Catanzaro, Italy) were electroporated into $1 \times 10^{6} \mathrm{D} 2.0 \mathrm{R}$ and D2A1 cells using the Amaxa Nucleofector (Lonza) according to the manufacturer's protocol. 
Knockdown of $\operatorname{Src}$ and $p 27$ using lentiviral particles expressing shRNAs is described in the Supplemental Methods.

\section{Proliferation assay}

Cells $\left(2 \times 10^{3}\right)$ were resuspended in $100 \mu \mathrm{l}$ DMEM $(1 \mathrm{~g} / \mathrm{l}$ glucose; Invitrogen $)$ plus $2 \%$ FBS and $2 \%$ Cultrex basement membrane extract (BME) (Trevigen) or $2 \%$ FBS plus BME and COL1, with or without inhibitors, respectively (final concentration of COL1: $2 \mathrm{mg} / \mathrm{ml}$ ), and were grown in triplicate in 96-well plates coated with $50 \mu \mathrm{l}$ BME or BME plus COL1. The number of viable cells was measured using the CellTiter-Glo One Solution Assay (Promega) according to the manufacturer's instructions.

\section{Caspase $3 / 7$ activity assay}

Caspase $3 / 7$ activity was performed using the Caspase-Glo 3/7 Assay (Promega) according to the manufacturer's protocol. A detailed description is provided in the Supplemental Methods.

\section{Real-time quantitative PCR}

RNA was extracted from 3D cultures using the TRIzol (Invitrogen) method according to the manufacturer's instructions. Real-time PCR was performed with $40 \mathrm{ng}$ RNA per sample in triplicate according to the SYBR green method (Bio-Rad). Details of the primer sequences are provided in the Supplemental Methods.

\section{Western blot analysis}

Western blotting was carried out as previously described (11). The antibodies used were: anti-Src (GD11; EMD Millipore), $\mathrm{pSrc}\left(\mathrm{Y}^{416}\right)$, $\mathrm{pFAK}\left(\mathrm{Y}^{576 / 577}\right)$, pERK1/2 ( $\left.\mathrm{T}^{202} / \mathrm{Y}^{204}\right)$, ERK1/2, p-p38 MAPK $\left(\mathrm{T}^{181} / \mathrm{Y}^{182}\right)$, p38 MAPK (Cell Signaling Technology), p27 (BD Biosciences), and $\beta$-actin (Sigma-Aldrich). Protein extractions from $3 \mathrm{D}$ cultures were performed as described (48). The SFK antibodies are listed in detail in the Supplemental Methods.

\section{Annexin V staining}

D2.0R cells were seeded on BME plus COL1 and treated with AZD0530, AZD6244, or their combination for 48 hours as described and extracted as previously described (48), with the use of EDTA-free trypsin. Cells were then stained with annexin $V$ and PI using the BD Pharmingen FITC Annexin V Apoptosis Detection Kit according to the manufacturer's protocol and analyzed by flow cytometry using an LSRFortessa cell analyzer (BD Biosciences).

\section{Cell cycle analysis}

After pulsing with BrdU for 1 hour, cells were extracted from matrices as described in ref. 48, processed as previously described (49), and analyzed by flow cytometry using a FACSCalibur flow cytometer (BD Biosciences).

\section{Immunofluorescence}

Cells were seeded in $3 \mathrm{D}$ culture as described above in 24-well plates with or without inhibitors. Cells with well coating were smeared on permafrost slides and processed as previously described (50). Slides were incubated with anti-p27 (c-19) (1/100; Santa Cruz Biotechnology Inc.) or anti-Ki67 $(1 / 1,000 ; \mathrm{Abcam})$ in $5 \% \mathrm{BSA}$ and Tris-buffered saline and Tween $20(0.05 \%)$ (TBST) overnight at $4{ }^{\circ} \mathrm{C}$ and were subsequently incubated with Alexa Fluor 594 goat anti-rabbit secondary antibodies for 1 hour, after which they were observed under a Nikon Eclipse E 800 fluorescence microscope (Nikon).

\section{Animal studies}

Pulmonary fibrosis. Pulmonary fibrosis was induced in 8-week-old female $\mathrm{CD} 1^{n u / n u}$ athymic mice (Charles River Laboratories) as previously described (51).
Experimental metastasis assays. Four weeks after infection, $1 \times 10^{6}$ D2.0R GFP cells, shNT D2.0R GFP cells, or shSrc D2.0R GFP cells (clones 3 and 4) in $100 \mu \mathrm{l}$ PBS were tail-vein injected into CD $1^{n u / n u}$ athymic female mice. Alternatively, $1 \times 10^{6}$ D2A1 GFP or shNT D2A1 GFP cells, or shSrc-1 D2A1 GFP or shSrc-2 D2A1 GFP cells were tailvein injected into 12 -week-old $\mathrm{CD} 1^{n u / n u}$ athymic female mice that had not received adenovirus.

\section{Inbibition of SFK with AZD0530}

Prevention regimen. Twenty-four hours after tail-vein injection of D2.0R GFP ( $n=15 /$ group) or D2A1-GFP cells ( $n=8 /$ group), mice were randomly divided into two groups and gavaged daily with $50 \mathrm{mg} / \mathrm{kg}$ body weight of AZD0530 or vehicle (mixture of $0.5 \%$ hydroxypropyl methyl cellulose plus $0.1 \%$ polysorbate) for 21 days.

Intervention regimen. Twenty-one days after tail-vein injection of D2.0R GFP cells, mice were randomly divided into two groups ( $n=10 /$ group) and gavaged daily as described above for an additional 21 days.

Combination regimen. Twenty-four hours after tail-vein injection of D2.0R GFP, mice were randomized into four groups, with group 1 receiving vehicle gavage $(n=5)$; group 2 receiving AZD0530 $(50 \mathrm{mg} / \mathrm{kg})(n=6)$; group 3 receiving AZD6244 $(25 \mathrm{mg} / \mathrm{kg})(n=5)$; and group 4 receiving AZD0530 $(50 \mathrm{mg} / \mathrm{kg})$ followed by AZD6244 $(25 \mathrm{mg} / \mathrm{kg}) 3$ hours later $(n=6)$.

Lung imaging. At the experimental endpoint, mice were euthanized and their lungs imaged by fluorescent single-cell, whole-organ microscopy (SCOM) imaging (Leica DM IRB) as previously described (11). Images of the total external surface of each lung were sequentially captured at $\times 10$ magnification and analyzed using Openlab software (PerkinElmer).

Ex vivo metastasis assay (PuMA). The PuMA assay was performed as previously described (24), and details of this assay are provided in the Supplemental Methods.

\section{Statistics}

All in vitro experiments were repeated at least three times, with representative blots and images shown. A 2-tailed Student's $t$ test was used for concentrations of MTS assays. All results are represented as the mean \pm SEM, with $P<0.05$ considered significant. Details of the statistical analyses of metastatic lesion measurements by SCOM have been previously described (11) and are provided in the Supplemental Methods.

\section{Study approval}

All mice were treated in accordance with the Guide for the Care and Use of Laboratory Animals (NIH publication no. 86-23, 1985) under an animal protocol (LCBG-011) approved by the IACUC of the National Cancer Institute (NCI).

\section{Acknowledgments}

This work was supported by the Intramural Research Program of the NIH, CCR, and NCI. We thank Glenn Merlino, Stuart Yuspa, Peter Blumberg, and Olga Aprelikova for useful discussions; Julie Foley and Norris Flagler for the collagen image analysis; and the Laboratory Animal Science Program and the FACS core facility at the NCI for excellent technical assistance.

Received for publication April 3, 2013, and accepted in revised form October 3, 2013.

Address correspondence to: Jeffrey E. Green, Laboratory of Cancer Biology and Genetics, National Cancer Institute, Building 37, Room 4054, 37 Convent Dr., Bethesda, Maryland 20892, USA. Phone: 301.435.5193; Fax: 301.496.8709; E-mail: jegreen@nih.gov. 
1. Siegel R, Naishadham D, Jemal A. Cancer statistics, 2012. CA Cancer J Clin. 2012;62(1):10-29.

2. Janni W, Rack B, Lindemann K, Harbeck N. Detection of micrometastatic disease in bone marrow: is it ready for prime time? Oncologist. 2005; 10(7):480-492.

3. Schmidt-Kittler O, et al. From latent disseminated cells to overt metastasis: genetic analysis of systemic breast cancer progression. Proc Natl Acad Sci US A. 2003;100(13):7737-7742.

4. Goodison S, et al. Prolonged dormancy and site-specific growth potential of cancer cells spontaneously disseminated from nonmetastatic breast tumors as revealed by labeling with green fluorescent protein. Clin Cancer Res. 2003;9(10 pt 1):3808-3814.

5. Aguirre-Ghiso JA. Models, mechanisms and clinical evidence for cancer dormancy. Nat Rev Cancer. 2007; 7(11):834-846

6. Lu P, Weaver VM, Werb Z. The extracellular matrix: a dynamic niche in cancer progression. J Cell Biol. 2012;196(4):395-406

7. Weaver VM, et al. Reversion of the malignant phenotype of human breast cells in three-dimensional culture and in vivo by integrin blocking antibodies. J Cell Biol. 1997;137(1):231-245.

8. Barkan D, Green JE, Chambers AF. Extracellular matrix: a gatekeeper in the transition from dormancy to metastatic growth. Eur J Cancer. 2010; 46(7):1181-1188

9. Morris VL, et al. Mammary carcinoma cell lines of high and low metastatic potential differ not in extravasation but in subsequent migration and growth. Clin Exp Metastasis. 1994;12(6):357-367.

10. Barkan D, et al. Inhibition of metastatic outgrowth from single dormant tumor cells by targeting the cytoskeleton. Cancer Res. 2008;68(15):6241-6250.

11. Barkan D, et al. Metastatic growth from dormant cells induced by a col-I-enriched fibrotic environment. Cancer Res. 2010;70(14):5706-5716.

12. Legate KR, Wickstrom SA, Fassler R. Genetic and cell biological analysis of integrin outside-in signaling. Genes Dev. 2009;23(4):397-418.

13. Zhang L, et al. c-Src expression is predictive of poor prognosis in breast cancer patients with bone metastasis, but not in patients with visceral metastasis. APMIS. 2012;120(7):549-557.

14. Anbalagan M, Moroz K, Ali A, Carrier L, Glodowski S, Rowan BG. Subcellular localization of total and activated Src kinase in African American and Caucasian breast cancer. PloS One. 2012;7(3):e33017.

15. Zhang XH-F, et al. Latent bone metastasis in breast cancer tied to Src-dependent survival signals. Cancer cell. 2009;16(1):67-78.

16. Myoui A, et al. C-SRC tyrosine kinase activity is associated with tumor colonization in bone and lung in an animal model of human breast cancer metastasis. Cancer Res. 2003;63(16):5028-5033.

17. Green TP, et al. Preclinical anticancer activity of the potent, oral Src inhibitor AZD0530. Mol Oncol. 2009; 3(3):248-261.

18. Lin TH, et al. Integrin-mediated activation of MAP kinase is independent of FAK: evidence for dual integrin signaling pathways in fibroblasts. $J$ Cell Biol. 1997;136(6):1385-1395.

19. Eralp Y, et al. MAPK overexpression is associated with anthracycline resistance and increased risk for recurrence in patients with triple-negative breast cancer. Ann Oncol. 2008;19(4):669-674.

20. Hulit J, et al. N-cadherin signaling potentiates mammary tumor metastasis via enhanced extracellular signal-regulated kinase activation. Cancer Res. 2007;67(7):3106-3116.

21. Catalanotti F, et al. Phase II trial of MEK inhibitor selumetinib (AZD6244) in patients with BRAFV600E/K-mutated melanoma. Clin Cancer Res. 2013;19(8):2257-2264.

22. Baselga J, et al. Phase I safety, pharmacokinetics, and inhibition of SRC activity study of saracatinib in patients with solid tumors. Clin Cancer Res. 2010; 16(19):4876-4883

23. Pegram MD, Silva OE, Higgins C, Tukia K, Stuart $\mathrm{M}$, Slingerland J. Phase IB pharmacokinetic (PK) study of Src kinase inhibitor AZD0530 plus anastrozole in postmenopausal hormone receptor positive $(\mathrm{HR}+)$ metastatic breast cancer $(\mathrm{MBC})$. J Clin Oncol. 2010;28(suppl):abstre13074.

24. Mendoza A, et al. Modeling metastasis biology and therapy in real time in the mouse lung.J Clin Invest. 2010;120(8):2979-2988

25. Aguirre-Ghiso JA, Estrada Y, Liu D, Ossowski L. ERK(MAPK) activity as a determinant of tumor growth and dormancy; regulation by $\mathrm{p} 38$ (SAPK). Cancer Res. 2003;63(7):1684-1695.

26. Lacroix M. Significance, detection and markers of disseminated breast cancer cells. Endocr Relat Cancer. 2006;13(4):1033-1067.

27. Mackay HJ, et al. A phase II trial of the Src kinase inhibitor saracatinib (AZD0530) in patients with metastatic or locally advanced gastric or gastro esophageal junction (GEJ) adenocarcinoma: a trial of the PMH phase II consortium. Invest New Drugs. 2012;30(3):1158-1163.

28. Elsberger B, et al. Is expression or activation of Src kinase associated with cancer-specific survival in ER-, PR- and HER2-negative breast cancer patients? Am J Pathol. 2009;175(4):1389-1397.

29. Morgan L, et al. Elevated Src kinase activity attenuates Tamoxifen response in vitro and is associated with poor prognosis clinically. Cancer Biol Ther. 2009; 8(16):1550-1558

30. Wilson GR, et al. Activated c-SRC in ductal carcinoma in situ correlates with high tumour grade, high proliferation and HER2 positivity. BrJ Cancer. 2006;95(10):1410-1414

31. Gucalp A, et al. Phase II trial of saracatinib (AZD0530), an oral SRC-inhibitor for the treatment of patients with hormone receptor-negative metastatic breast cancer. Clin Breast Cancer. 2011; 11(5):306-311

32. Raviraj V, Zhang H, Chien $\mathrm{H}$, Cole L, Thompson EW, Soon L. Dormant but migratory tumour cells in desmoplastic stroma of invasive ductal carcinomas. Clin Exp Metastasis. 2012;29(3):273-292.

33. Chu I, et al. p27 phosphorylation by Src regulates inhibition of cyclin E-Cdk2. Cell. 2007;128(2):281-294.

34. Sánchez-Bailón MP, et al. Src kinases catalytic activity regulates proliferation, migration and invasiveness of MDA-MB-231 breast cancer cells. Cell Signal. 2012;24(6):1276-1286.

35. Le X-F, et al. The role of p27(Kip1) in dasatinib-en- hanced paclitaxel cytotoxicity in human ovarian cancer cells. J Natl Cancer Inst. 2011;103(18):1403-1422.

36. Cox BD, Natarajan M, Stettner MR, Gladson CL. New concepts regarding focal adhesion kinase promotion of cell migration and proliferation. J Cell Biochem. 2006;99(1):35-52.

37. Ciccimaro E, Hanks SK, Blair IA. Quantification of focal adhesion kinase activation loop phosphorylation as a biomarker of Src activity. Mol Pharm. 2009; 75(3):658-666

38. Wu FY, et al. Reduction of cytosolic p27(Kip1) inhibits cancer cell motility, survival, and tumorigenicity. Cancer Res. 2006;66(4):2162-2172.

39. McAllister SS, Becker-Hapak M, Pintucci G, Pagano M, Dowdy SF. Novel p27(kip1) C-terminal scatter domain mediates Rac-dependent cell migration independent of cell cycle arrest functions. $\mathrm{Mol}$ Cell Biol. 2003;23(1):216-228.

40. Lee EY, Jeon MJ, Yang W, Shin I. Effect of p27 on motility of MDA-MB-231 breast cancer cells. Oncol Rep. 2009;21(6):1621-1625.

41. Wendt MK, Taylor M, Schiemann BJ, Schiemann WP. Down-regulation of epithelial cadherin is required to initiate metastatic outgrowth of breast cancer. Mol Biol Cell. 2011;22(14):2423-2435.

42. Imamura $T$, Hikita $A$, Inoue $Y$. The roles of TGF- $\beta$ signaling in carcinogenesis and breast cancer metastasis. Breast Cancer. 2012;19(2):118-124.

43. Ferguson J, Arozarena I, Ehrhardt M, Wellbrock C. Combination of MEK and SRC inhibition suppresses melanoma cell growth and invasion. Oncogene. 2012;32(1):86-96.

44. Miller FR, Miller BE, Heppner GH. Characterization of metastatic heterogeneity among subpopulations of a single mouse mammary tumor: heterogeneity in phenotypic stability. Invasion Metastasis. 1983; 3(1):22-31.

45. Naumov GN, et al. Persistence of solitary mammary carcinoma cells in a secondary site: a possible contributor to dormancy. Cancer Res. 2002;62(7):2162-2168.

46. Aslakson CJ, Miller FR. Selective events in the metastatic process defined by analysis of the sequential dissemination of subpopulations of a mouse mammary tumor. Cancer Res. 1992;52(6):1399-1405.

47. Khanna C, et al. The membrane-cytoskeleton linker ezrin is necessary for osteosarcoma metastasis. Nat Med. 2004;10(2):182-186.

48. Johnson KR, Leight JL, Weaver VM. Demystifying the effects of a three-dimensional microenvironment in tissue morphogenesis. Methods Cell Biol. 2007;83:547-583.

49. Cariou S, Donovan JC, Flanagan WM, Milic A, Bhattacharya N, Slingerland JM. Down-regulation of p21WAF1/CIP1 or p27Kip1 abrogates antiestrogenmediated cell cycle arrest in human breast cancer cells. Proc Natl Acad Sci U S A. 2000;97(16):9042-9046.

50. Lee GY, Kenny PA, Lee EH, Bissell MJ. Three-dimensional culture models of normal and malignant breast epithelial cells. Nat Methods. 2007; 4(4):359-365.

51. Sime PJ, Xing Z, Graham FL, Csaky KG, Gauldie $\mathrm{J}$. Adenovector-mediated gene transfer of active transforming growth factor-beta 1 induces prolonged severe fibrosis in rat lung. J Clin Invest. 1997; 100(4):768-776. 